\title{
Role of Gas6 in erythropoiesis and anemia in mice
}

\author{
Anne Angelillo-Scherrer, ${ }^{1}$ Laurent Burnier, ${ }^{1}$ Diether Lambrechts, ${ }^{2}$ Richard J. Fish, ${ }^{3}$ Marc Tjwa, ${ }^{2}$ \\ Stéphane Plaisance, ${ }^{2}$ Rocco Sugamele, ${ }^{1}$ Maria DeMol, ${ }^{2}$ Eduardo Martinez-Soria, ${ }^{4}$ \\ Patrick H. Maxwell, ${ }^{5}$ Greg Lemke, ${ }^{6}$ Stephen P. Goff, ${ }^{7}$ Glenn K. Matsushima, ${ }^{8}$ \\ H. Shelton Earp, ${ }^{9}$ Marc Chanson, ${ }^{10}$ Désiré Collen, ${ }^{2}$ Shozo Izui, 4 \\ Marc Schapira, ${ }^{1}$ Edward M. Conway, ${ }^{2}$ and Peter Carmeliet $^{2}$
}

${ }^{1}$ Service and Central Laboratory of Hematology, Centre Hospitalier Universitaire Vaudois, University Hospital Center and University of Lausanne, Lausanne, Switzerland. ${ }^{2} \mathrm{VIB}$, Department of Transgene Technology and Gene Therapy, Leuven, Belgium and Center for Transgene Technology and Gene Therapy, KU Leuven, Leuven, Belgium. ${ }^{3}$ Division of Angiology and Hemostasis and ${ }^{4}$ Department of Pathology and Immunology, University Medical Center, Geneva, Switzerland. ${ }^{5}$ Renal Section, Imperial College of London, Hammersmith Campus, London, United Kingdom. ${ }^{6}$ Salk Institute for Biological Studies, La Jolla, California, USA. ${ }^{7}$ Department of Biochemistry and Molecular Biophysics, Columbia University Medical Center, New York, New York, USA. 8UNC Neuroscience Center and 'Lineberger Comprehensive Cancer Center, University of North Carolina, Chapel Hill, North Carolina, USA. ${ }^{10}$ Department of Pediatrics, University Hospital and Faculty of Medicine, Geneva, Switzerland.

\begin{abstract}
Many patients with anemia fail to respond to treatment with erythropoietin (Epo), a commonly used hormone that stimulates erythroid progenitor production and maturation by human BM or by murine spleen. The protein product of growth arrest-specific gene $6(\mathrm{Gas} 6)$ is important for cell survival across several cell types, but its precise physiological role remains largely enigmatic. Here, we report that murine erythroblasts released Gas6 in response to Epo and that Gas6 enhanced Epo receptor signaling by activating the serine-threonine kinase Akt in these cells. In the absence of Gas6, erythroid progenitors and erythroblasts were hyporesponsive to the survival activity of Epo and failed to restore hematocrit levels in response to anemia. In addition, Gas6 may influence erythropoiesis via paracrine erythroblast-independent mechanisms involving macrophages. When mice with acute anemia were treated with Gas6, the protein normalized hematocrit levels without causing undesired erythrocytosis. In a transgenic mouse model of chronic anemia caused by insufficient Epo production, Gas6 synergized with Epo in restoring hematocrit levels. These findings may have implications for the treatment of patients with anemia who fail to adequately respond to Epo.
\end{abstract}

\section{Introduction}

Anemia results from insufficient production, excessive destruction, or loss of $r b c$ (1). By limiting the capacity of the blood to carry oxygen, anemia causes tissue hypoxia and may lead to severe cardiovascular, neurological, and musculoskeletal malfunction. In response to anemia, new rbc are generated. This process of erythropoiesis relies on the commitment of hematopoietic stem cells to differentiate into erythroblasts, which subsequently mature into circulating rbc. While erythropoiesis is regulated by several cytokines, erythropoietin (Epo) is a key factor that induces the maturation and proliferation of burst-forming unit and CFU erythroid progenitors (BFU-Es and CFU-Es, respectively) and early erythroblasts (2). Epo is also an important survival factor for these cells, as deprivation of Epo leads to apoptosis (3). Besides regulating erythropoiesis in baseline conditions, Epo also induces a compensatory erythropoietic response to reduced tissue oxygen tension (hypoxia) and anemia. In humans, Epo promotes recovery from anemia by stimulating the generation of erythroid progenitors in the BM, while in the mouse, Epo enhances erythropoiesis primarily in the spleen (4).

Recombinant Epo is widely used to treat anemia associated with numerous disorders such as chronic renal failure, myelodysplastic

Nonstandard abbreviations used: BFU-E, burst-forming unit erythroid; CFU-E, CFU erythroid; Epo, erythropoietin; EpoR, Epo receptor; Gas6, growth arrest-specific gene 6; Gas6R, Gas6 receptor; PHZ, phenylhydrazine; rGas6, recombinant Gas6.

Conflict of interest: P.H. Maxwell is a consultant and director of ReOx Ltd. Citation for this article: J. Clin. Invest. 118:583-596 (2008). doi:10.1172/JCI30375. syndromes, cancer, and rheumatoid arthritis (5-8). However, Epo is not always effective, with many individuals being refractory to even high doses $(9,10)$. Approximately $10 \%$ of patients with endstage renal disease are hyporesponsive, over $50 \%$ of patients with cancer have an inadequate response to Epo, and only $20 \%-25 \%$ of patients with myelodysplastic syndromes respond favorably $(6,9,10)$. Although inflammation, iron and vitamin deficiency, inadequate dialysis, aluminium toxicity, and hyperparathyroidism impair the response to Epo, the molecular mechanisms of the resistance to Epo remain largely obscure $(9,11)$.

We therefore explored whether other factors might stimulate erythropoiesis. We considered growth arrest-specific gene 6 (Gas6) - acting via its receptor tyrosine kinases, the Gas6 receptors (Gas6Rs) Tyro3, Axl, and Mertk - as a likely candidate, because each of its receptors is expressed in hematopoietic tissue, megakaryocytes, myelomonocytic precursors, and BM stromal cells (12-15). Gas6 is a secreted vitamin K-dependent protein that interacts with phospholipid membranes via its $\gamma$-carboxyglutamic acid-containing tail and binds its receptors via its carboxyterminal globular domain (16-19). This leads to further intracellular signaling, including activation of the PI3K and Akt pathways $(20,21)$. Gas6 has been implicated in reversible cell growth arrest, survival, proliferation, cell adhesion, and hemostasis (reviewed in ref. 22). Apart from its expression pattern, the biological role of Gas6 in hematopoiesis remains largely unknown. Conflicting lines of evidence suggest that it lacks mitogenic activity for hematopoietic progenitors or stromal fibroblasts (12), whereas in vitro overexpression of Gas6 in fibroblasts facilitates the generation of hematopoietic CFUs (23). 
A
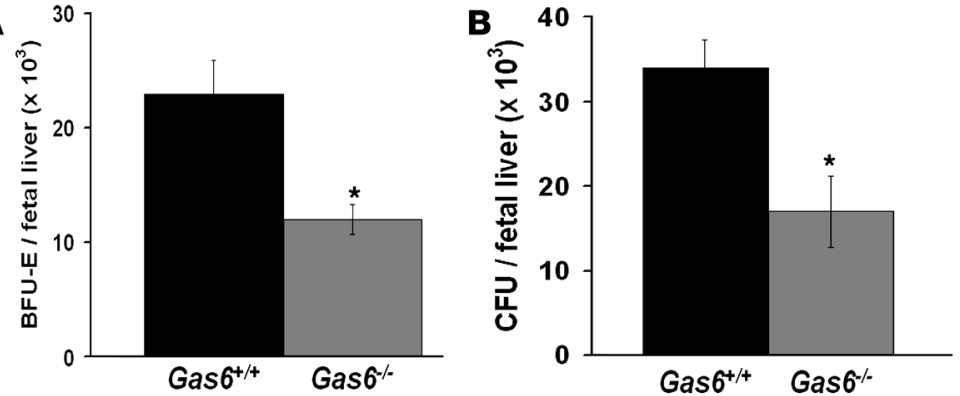

C

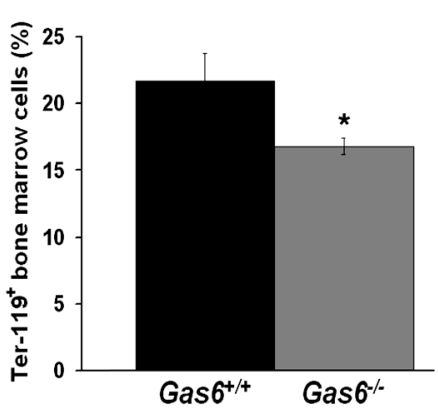

$\mathbf{E}$

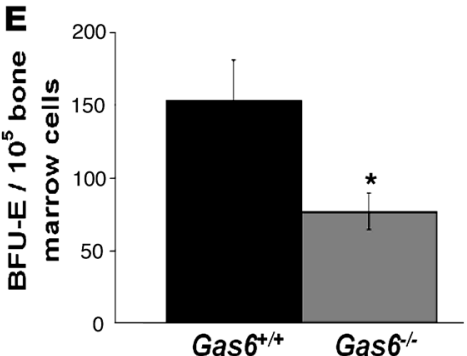

D
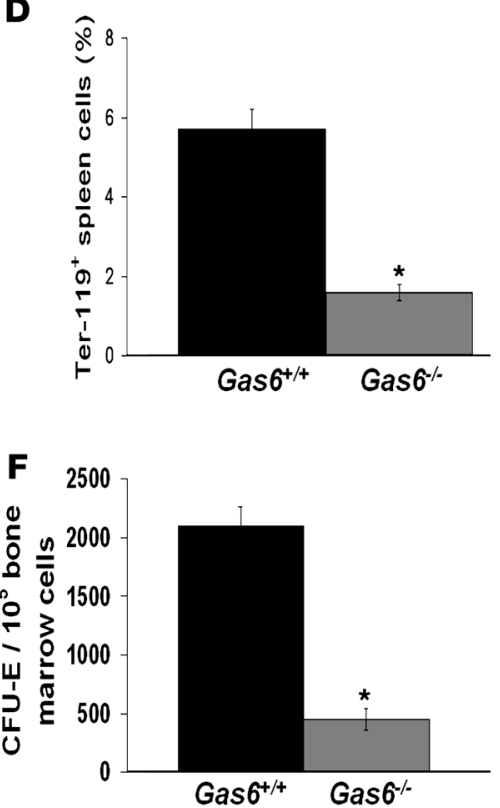

Figure 1

Defects in the erythroid lineage of WT and Gas6-/- mice. (A and B) Reduced number of erythroid progenitors in fetal liver of Gas6 $^{-/-}$mice, analyzed as the number of BFU-Es (A) or CFU-Es (B). $n=6$ per group. ${ }^{*} P<0.05$. (C and D) Reduced percentage of Ter-119+ nucleated cells (erythroblasts) in adult BM $\left(\mathbf{C} ;{ }^{*} P=0.02\right)$ and spleen $\left(\mathbf{D} ;{ }^{*} P<0.02\right)$ in Gas6 $^{-1-}$ mice. The erythroblasts were quantified as the percent of all nucleated cells by flow cytometry. $n=9$ per group. ( $E$ and $\mathbf{F})$ Reduced number of erythroid progenitors in the BM of adult Gas6- ${ }^{-1-}$ mice, analyzed as the number of BFU-Es (E) or CFU-Es (F). $n=8$ per group. ${ }^{*} P<0.05$. Values are mean \pm SEM.

Because of the decreased erythroid reserves in the embryo, we expected the defect to manifest in adult $\mathrm{Gas}^{-{ }^{--}}$mice by lower hematocrit levels. Surprisingly, Gas $6^{-/-}$mice had normal hematocrit levels under baseline conditions (WT, 48.8\% $\pm 1.0 \%$; Gas $6^{-/}, 49.1 \% \pm 3.9 \%$; $n=12 ; P=\mathrm{NS}$ ).

Indices and morphology of rbc were comparable in WT and $\mathrm{Gas}^{-/-}$mice. BM cytological examination showed that $\mathrm{Gas}^{-/-}$erythroid cells were present at all maturation stages and did not display signs of dysplasia (data not shown). However, reticulocyte counts were lower in adult $\mathrm{Gas}^{-/-}$than WT mice (WT, $2.5 \% \pm 0.1 \%$; Gas6 $\left.6^{-/-}, 1.3 \% \pm 0.1 \% ; n=8 ; P<0.01\right)$. The reduced reticulocyte levels in adult $\mathrm{Gas}^{-/-}$mice were not attributable to a defect in reticulocyte maturation (Supplemental Note 1; supplemental material available online with this article; doi:10.1172/JCI30375DS1), but rather to a partial depletion of erythroid reserves in both BM and spleen. Indeed, flow cytometry of Ter- $119^{+}$cells revealed the presence of fewer erythroblasts in BM and spleen of $\mathrm{Gas}^{-/-}$mice (Figure 1, C and D). Fewer

In this study, we identified what we believe to be a novel role for Gas6 in amplifying the erythropoietic response to Epo and characterized its therapeutic potential in mouse models of acute and chronic anemia. These findings warrant further investigation of the therapeutic potential of Gas 6 for the treatment of patients who are hyporesponsive or resistant to Epo.

\section{Results}

Deficits in the erythroid lineage in Gas6 $6^{-/}$embryos. We previously reported that adult $\mathrm{Gas}^{-/-}$mice have a platelet dysfunction but otherwise appear phenotypically normal (24). However, development of $\mathrm{Gas}^{-1-}$ embryos was not previously investigated. Here we found that livers of $\mathrm{Gas}^{-/-}$embryos were smaller at E13.5 compared with WT embryos, while the other organs appeared normal. Because fetal liver is a site of embryonic erythropoiesis (25), we further assessed the role of Gas 6 in this process. By flow cytometry, we found fetal livers to contain fewer erythroblasts in $\mathrm{Gas}^{-/-}$than WT embryos (Ter-119+ erythroblasts per liver, WT, $8.2 \pm 0.11 \times 10^{6}$; Gas6-/- $\left.3.9 \pm 0.05 \times 10^{6} ; n=6 ; P<0.01\right)$. When using in vitro clonogenic assays, fetal livers of $\mathrm{Gas}^{-/-}$embryos contained half the number of BFU-Es and CFU-Es as those present in WT embryos (Figure 1, A and B). Thus, absence of Gas6 leads to a deficit in embryonic erythroid precursor cells.

Defects in the erythroid lineage in adult $\mathrm{Gas}^{6^{-/}}$mice. We next examined whether erythropoiesis was impaired in adult $\mathrm{Gas}^{-/-}$mice.
Ter- $119^{+}$erythroblasts were also detected by immunostaining in the spleen (data not shown). Furthermore, the spleens weighed approximately $20 \%$ less in Gas6 $6^{-/-}$mice (WT, $113 \pm 3 \mathrm{mg}$; Gas $6^{-/-}$, $91 \pm 15 \mathrm{mg} ; n=33 ; P<0.01)$.

The erythropoietic defect in adult $\mathrm{Gas}^{-/-}$mice could be attributable to a defect in erythroid progenitor cells, as fewer BFU-E and CFU-E colonies developed after plating out equal numbers of BM cells (Figure 1, E and F). Supplementation of recombinant Gas6 (rGas6; $400 \mathrm{ng} / \mathrm{ml}$ ) to the culture medium rescued the impaired formation of BFU-E colonies in $\mathrm{Gas}^{-1-}$ mice to levels approximating those found in WT mice (BFU-E colonies per $10^{5} \mathrm{BM}$ cells, WT, $139 \pm 4$; WT plus rGas6, $143 \pm 6$; Gas6 $^{-1-}, 59 \pm 3$; Gas6 $^{-/-}$plus rGas6, $125 \pm 4 ; n=10, P<0.001)$. These data suggest that rGas 6 regulates erythropoiesis, at least in part, by increasing the plating efficacy of Gas $6^{-/-}$erythroid progenitors (see below for effects of Gas6 on erythroblast adhesion). Thus, in the absence of Gas6, fewer erythroid progenitors and precursors were generated in the adult BM.

Reduced clearance rate of senescent rbc in $\mathrm{Gas}^{-/-}$mice. Our data demonstrate that an abnormal (accelerated) rate of reticulocyte maturation could not explain the normal hematocrit levels despite the reduced reticulocyte counts. We therefore analyzed whether rbc have a longer lifespan in $\mathrm{Gas}^{-/-}$mice. Indeed, the clearance rate of biotinylated $\mathrm{rbc}$ was lower in $\mathrm{Gas} 6^{-/-}$than WT mice, as evidenced by the higher percentage of residual biotinylated rbc at 30 days after injection (WT, $19 \% \pm 3 \%$; Gas6 $6^{--}, 31 \% \pm 4 \% ; n=12 ; P<0.05$ ). The 
A

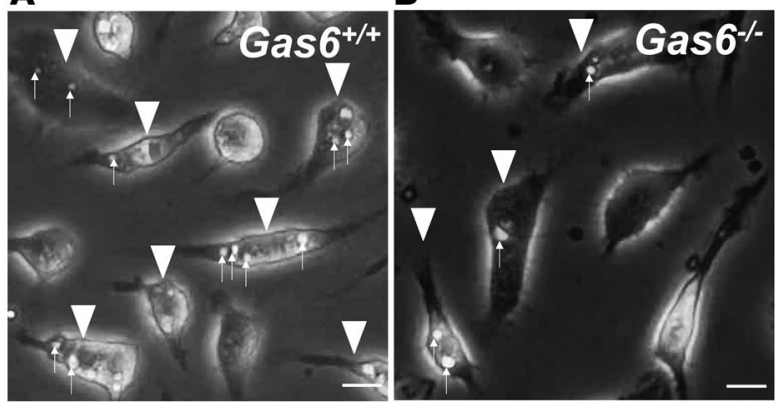

C

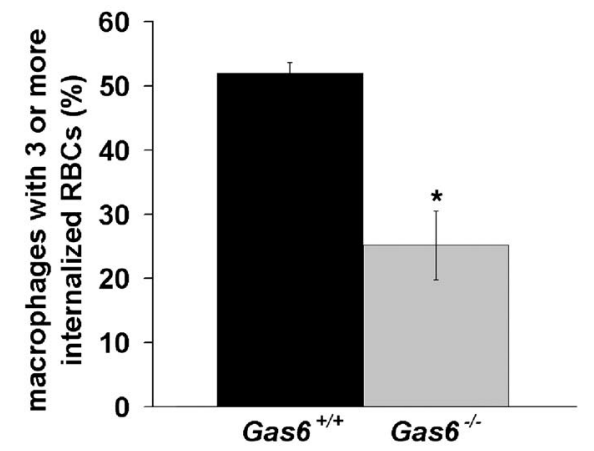

subpopulation of rbc labeled with both biotin and annexin $\mathrm{V}$ was considered senescent $\mathrm{rbc}(26)$. We found that a higher number of senescent rbc circulated in $\mathrm{Gas}^{-/-}$than WT mice. The percentage of biotinylated $\mathrm{rbc}$ that were annexin $\mathrm{V}^{+}$after 30 days was $0.11 \% \pm 0.03 \%$ in WT mice versus $0.31 \% \pm 0.11 \%$ in $\mathrm{Gas}^{-/-}$mice $(n=12 ; P<0.05)$. These data indicate that $\mathrm{rbc}$ in $\mathrm{Gas}^{-/-}$mice had a longer lifespan, because senescent $\mathrm{rbc}$ were not efficiently cleared.

Gas 6 has been previously documented to form a bridge between phosphatidylserine (exposed on apoptotic or senescent cells) and Gas6Rs on macrophages, which then engulf and clear these cells (27). To investigate whether $\mathrm{Gas}^{-/-}$macrophages similarly engulf senescent rbc less efficiently, we treated rbc with phenylhydrazine (PHZ) to expose phosphatidylserine on their surface (28) and incubated them with adherent macrophages. The number of macrophages that engulfed 3 or more rbc was reduced by more than $50 \%$ in Gas $6^{-/-}$mice (Figure 2). Similar findings were obtained when using Gas $6^{-/-}$mice on $100 \%$ C57BL/ 6 and mixed 50\% $129 / \mathrm{Sv} \times 50 \%$ Swiss genetic backgrounds (data not shown). Thus, despite an underrepresentation of erythroid progenitors and precursor cells, hematocrit levels under baseline conditions were preserved in $\mathrm{Gas}^{-{ }^{--}}$mice, because of a prolongation of $\mathrm{Gas}^{-/-} \mathrm{rbc}$ survival due to the reduced engulfment of senescent $\mathrm{rbc}$ by macrophages.

Impaired erythropoietic response to acute anemia in $\mathrm{Gas}^{-/-}$mice. In adulthood, only a minimal degree of erythropoiesis is required to preserve hematocrit levels during baseline conditions. However, in response to anemia, erythropoiesis is rapidly increased in order to restore hematocrit levels. We therefore assessed whether the erythropoietic defect in $\mathrm{Gas}^{-/-}$mice might impair their response to restore hematocrit levels when challenged with acute anemia. Two models of hemolytic anemia were used. In the first model, PHZ was injected intraperitoneally to cause rapid hemolysis. Hematocrit levels, reticulocyte counts, and spleen size were monitored over the ensuing 10 days (Figure 3, A and B). From day 3 to day 9, after $2 \mathrm{PHZ}$ injections on days 0 and 1 , hematocrit levels were significantly lower in $\mathrm{Gas}^{-/-}$than WT mice (Figure 3A). In WT mice,

\section{Figure 2}

Senescent rbc engulfment by WT and $\mathrm{Gas}^{-/-}$macrophages. WT and $\mathrm{Gas6}^{-/-}$mouse rbc were treated with $\mathrm{PHZ}$ to expose phosphatidylserine on their surface and then incubated with primary adherent BM-derived macrophages. (A and B) Macrophages in phase-contrast illumination are indicated by arrowheads; engulfed rbc are denoted by arrows. Phagocytosis was impaired in $\mathrm{Gas6}^{-1-}$ mice (B) compared with WT mice (A). Scale bars: $20 \mu \mathrm{m}$. (C) The number of macrophages with 3 or more internalized rbc was reduced in $\mathrm{Gas}^{-/-}$mice compared with WT mice $\left(n=250\right.$; mean \pm SEM; $\left.{ }^{*} P=0.001\right)$. Data were reproduced in $100 \%$ C57BL/6 background (not shown).

hematocrit levels returned to normal by day 6 ; in contrast, hematocrit levels in $\mathrm{Gas}^{-/-}$mice failed to recover until day 10 after PHZ, indicating that erythropoietic response was impaired and delayed in the absence of Gas6. Similar findings were obtained when using Gas $6^{-/-}$mice on mixed 50\% $129 / \mathrm{Sv} \times 50 \%$ Swiss (Figure 3A) and $100 \%$ C57BL/ 6 genetic backgrounds (Supplemental Figure 1).

Because erythropoiesis in response to anemia occurs primarily in the spleen in mice (4), we measured the spleen weight as an indirect parameter of erythropoiesis. By day 3 after $\mathrm{PHZ}$ injection, the spleen was already enlarged more in WT than $\mathrm{Gas}^{-/-}$mice (before and 3 days after PHZ, WT, $113 \pm 3$ and $270 \pm 7 \mathrm{mg}$; Gas $^{-/}$, $91 \pm 15$ and $177 \pm 12 \mathrm{mg} ; n=10-21 ; P<0.01)$. By day 6 , the difference was more apparent (WT, $620 \pm 45 \mathrm{mg}$; Gas $6^{-/}, 410 \pm 36 \mathrm{mg} ; P<0.01$ ); spleen weight increased 5.5 -fold in WT mice but only 4.5 -fold in $\mathrm{Gas}^{-/-}$mice. In addition, the reticulocyte response was delayed in Gas $6^{-1-}$ mice, as evidenced by the 2 -fold lower reticulocyte counts in Gas $6^{-1-}$ than WT mice at day 6 after PHZ (Figure 3B). Thus, the absence of Gas 6 did not cause anemia spontaneously, but impaired the erythropoietic recovery in conditions of acute anemia.

In the second model, immune hemolytic anemia was induced by administration of the anti-mouse rbc antibody 34-3C (29). This antibody caused a comparable degree of hemolysis in both genotypes (Supplemental Note 2). As in the PHZ model, hematocrit levels and reticulocyte counts recovered more slowly in $\mathrm{Gas}^{-1-}$ than WT mice (Figure 3, C and D). When analyzing the number of erythroblasts in the spleen by flow cytometry, we found that $\mathrm{Gas} 6^{-/-}$mice also contained fewer Ter- $119^{+}$cells at day 4 after induction of anemia (WT, $45 \% \pm 7 \%$; $\mathrm{Gas}^{-/}, 26 \% \pm 10 \%$; $n=10$; $P<0.05)$. Thus, also in this model, after induction of acute anemia, the $\mathrm{Gas}^{-/-}$mice were not able to achieve a normal hematocrit as rapidly as WT mice.

Role of Gas6 in BM erythropoiesis. In humans, erythropoiesis in response to anemia is induced in the spleen and in the BM. This is in contrast to mice, where erythropoiesis under similar conditions occurs primarily in the spleen, although the BM can also contribute when splenic function is compromised (4). To assess how relevant our genetic findings in the mouse might be for humans, we studied whether Gas6 also regulated erythropoiesis in the BM in response to anemia after removal of the spleen. As expected, splenectomy itself retarded the erythropoietic recovery in WT mice, and hematocrits only approximated normal levels by day 10 after PHZ injections on day 0 and 1 (Figure 3E). Notably, hematocrit levels were consistently lower in splenectomized $\mathrm{Gas}^{-/-}$mice and were still not completely restored by day 10 after PHZ (Figure 3E). This is also in contrast to the full recovery in hematocrit achieved at day 6 and sustained through day 10 by PHZ-treated nonsplenectomized Gas $6^{-/}$mice (Figure 3A). From these findings, we conclude that Gas6 regulates the erythropoietic response to acute anemia both in spleen and in BM. 

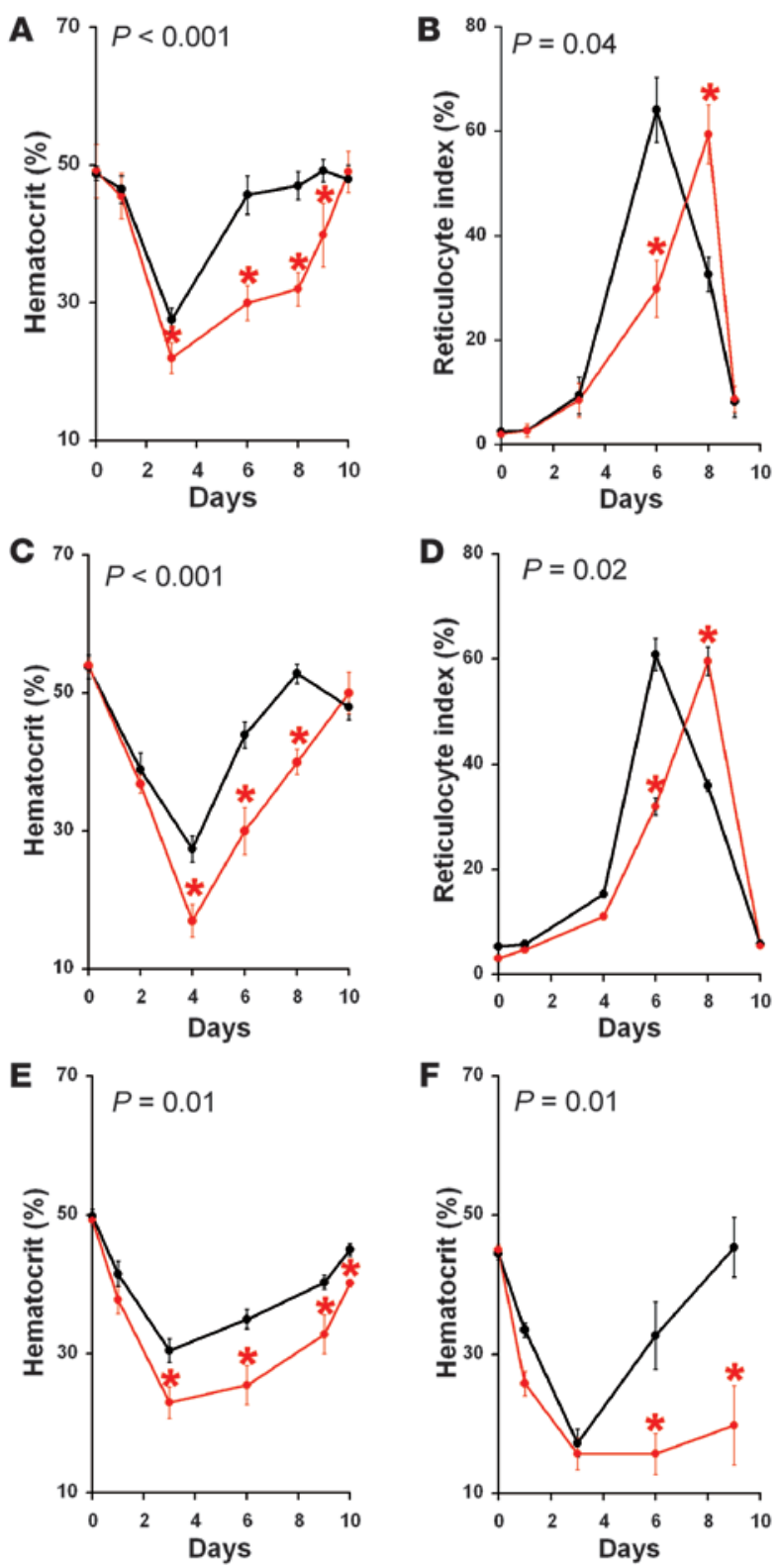

Impaired survival of $\mathrm{Gas}^{-/-}$mice in acute anemia. Anemia may be life-threatening when hematocrit levels acutely drop to low levels, particularly in clinical settings where cardiac reserves are jeopardized (30). To further assess the possible preclinical relevance of the erythropoietic defect in the Gas $6^{-1-}$ mice, we challenged mice with a more severe form of acute anemia by injecting PHZ on 3 consecutive days (days 0,1 , and 2 ). In both genotypes, hematocrit levels dropped below $20 \%$ by day 3 (Figure 3F). All WT mice were capable of restoring their hematocrit levels by day 9 and survived for the entire period. In contrast, up to two-thirds of the Gas $6^{-/-}$ mice died from day 3 onward; we sampled blood whenever possible from preterminal mice and found that all had extremely diminished hematocrit levels (mean, 11\%). Thus, Gas $6^{-/-}$mice remained significantly more anemic than did WT mice (Figure 3F). Even those few mice that survived the challenge throughout the period of observation remained anemic. Thus, Gas 6 confers erythropoietic protection against life-threatening acute anemia.

\section{Figure 3}

Erythropoietic response to acute anemia in WT (black lines) and Gas6-/(red lines) mice. $P$ values above the graphs denote overall genotypic difference during the entire period of analysis, as analyzed by ANOVA for repeated measurement statistics. ${ }^{*} P<0.05$, standard $t$ test. (A-D) Impaired erythropoiesis in $\mathrm{Gas6}^{-1-}$ compared with WT mice in response to $\mathrm{PHZ}$-induced hemolytic anemia. (A and B) Hematocrit levels (A) and reticulocyte indices (B) after $2 \mathrm{PHZ}$ injections on days 0 and 1 $(n=8)$. Data were reproduced in $100 \%$ C57BL/6 background (Supplemental Figure 1). (C and D) Impaired erythropoiesis in $\mathrm{Gas6}^{-/-}$mice in response to autoimmune hemolytic anemia, induced by intraperitoneal injection of the 34-3C anti-mouse rbc monoclonal antibody (200 $\mu \mathrm{g}$ on day 0). (C) Hematocrit levels. (D) Reticulocyte indices $(n=8)$. (E) Impaired erythropoiesis in Gas6 $^{-/-}$splenectomized mice in response to injections of $\mathrm{PHZ}$ injections on days 0 and $1(n=6)$. (F) Hematocrit levels in $\mathrm{Gas}^{-/-}$and WT mice after PHZ injections on days 0,1 and 2 $(n=6)$. A hematocrit value of $11 \%$ (the lowest detectable hematocrit level in preterminal mice) was assigned to the mice that died in the course of the experiment. Values are mean \pm SEM.

Erythroblasts express the Gas6Rs Tyro3, Axl, and Mertk. We examined the mechanisms by which Gas 6 mediates an erythropoietic response. For erythroblasts to respond directly to Gas6, they should express 1 or more of the Gas6Rs: Tyro3, Axl, and Mertk. We therefore analyzed whether Gas6Rs were expressed by splenic erythroblasts under steady-state conditions and 6 days after PHZ. Ter- $119^{+}$splenic erythroblasts from WT and Gas $6^{-/-}$mice were isolated, and quantitative RT-PCR analysis was used to measure transcript mRNA levels of each receptor, normalized to $\beta$-actin transcript levels. Transcripts of all the Gas6Rs - Tyro3, Axl, and Mertk - were expressed by erythroblasts of WT and Gas $6^{-/-}$mice. Accumulation of each Gas6R mRNA did not differ, however, between the 2 genotypes under steady-state conditions (Table 1), nor did the specific mRNA levels increase in response to administration of PHZ 6 days earlier (data not shown).

To provide more direct evidence that erythroblasts express the Gas6Rs at the protein level, we immunoblotted lysates of isolated Ter- $119^{+}$splenic erythroblasts. SDS gels were loaded with equivalent amounts of protein. As expected, both Axl and Mertk were indeed detectable in splenic erythroblasts, but not in $A x^{-/-}$or Mertk ${ }^{k d}$ splenic erythroblasts, respectively (Figure 4A). Expression levels of Axl and Mertk did not increase in erythroblasts that were isolated 6 days after PHZ treatment of WT mice (data not shown). Available anti-Tyro3 antibodies were not able to immunodetect denatured Tyro3 by Western blotting. As an alternative, we used a sensitive ELISA and found measurable levels of Tyro3 protein in

\section{Table 1}

Gene expression of Gas6Rs Tyro3, Axl, and Mertk in spleen erythroblasts

\begin{tabular}{|c|c|c|}
\hline & \multicolumn{2}{|c|}{ No. copies per $1,000 \beta$-actin copies } \\
\hline & WT & Gas6- $^{-/}$ \\
\hline Tyro3 & $49.5 \pm 9.5$ & $57.8 \pm 7.2$ \\
\hline$A \times I$ & $51.5 \pm 14.5$ & $53.3 \pm 6.5$ \\
\hline Mertk & $15.3 \pm 1.0$ & $15.3 \pm 2.5$ \\
\hline
\end{tabular}


A
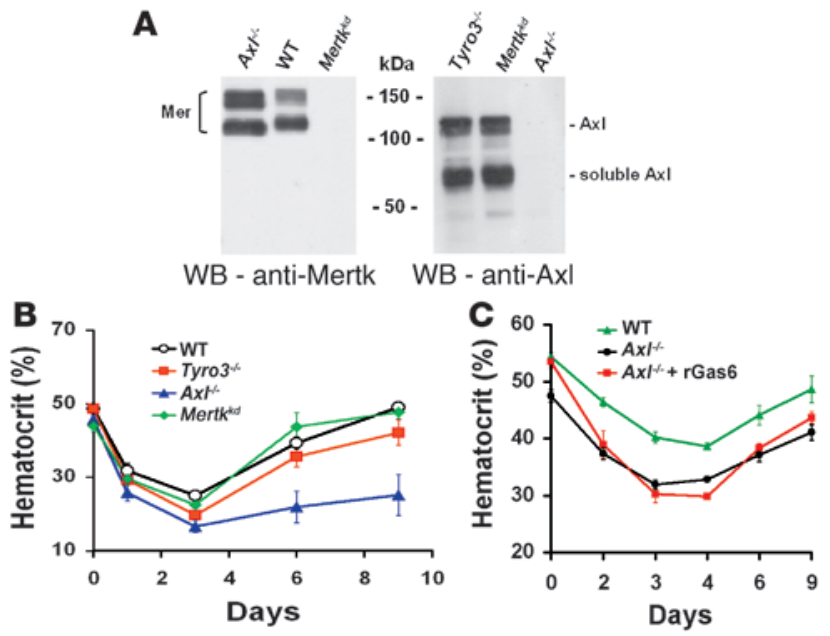

WT splenic erythroblast extracts $(0.87 \pm 0.12 \mathrm{ng} / \mathrm{mg}$ erythroblast extracts, $n=6$ ), but none in Tyro $3^{-/}$splenic erythroblast extracts, as expected. Thus, all 3 Gas6Rs are expressed by splenic erythroblasts and are candidates to mediate the erythropoietic activity of Gas6.

Axl mediates the erythropoietic response of Gas6. To provide direct evidence for a functional role of Gas6Rs in the erythropoietic response to acute anemia, we challenged Gas6R-deficient mice with PHZ to induce hemolytic anemia. Under baseline conditions, Tyro3 $3^{-1-}$, $A x l^{-/}$, and $\mathrm{Mertk}^{k d}$ mice had normal hematocrit levels. Induction of hemolytic anemia in mice with Gas6R gene inactivations and their WT counterparts was achieved by injections of PHZ on days 0 and 1 . After administration of PHZ, all WT and Mertk $k^{k d}$ mice recovered from the acute anemic stress with comparable hematocrit levels (Figure 4B). Most Tyro3 $3^{--}$mice were also capable of recovering from anemia with hematocrit levels similar to those of WT mice (Figure 4B); only $17 \%$ of Tyro $3^{-/}$mice died because of low hematocrit levels. In sharp contrast, hematocrit dropped to extremely low levels (11\%) in $A x l^{-/-}$mice. In fact, $56 \%$ of the $\mathrm{Axl}^{\mathrm{l}^{-/}}$mice failed to restore their hematocrit levels and died 3 or more days after the second PHZ injection (Figure 4B). Surviving $A x^{-/-}$mice also had lower hematocrit levels and were not able to fully recover from anemia (hematocrit nadir, WT, $25 \% \pm 1.4 \% ; A x l^{-/}, 19.5 \% \pm 1.6 \%$ ). Consistent herewith, reticulocyte counts at day 6 were comparable in WT, Tyro $3^{--}$, and Mertk $k^{k d}$ mice (data not shown), but lower in $\mathrm{Axt}^{-/}$mice (WT, 83\% $\pm 8 \% ; A x l^{--}, 47 \% \pm 6 \% ; n=4 ; P<0.05$ ).

The data indicates that in the response to hemolytic anemia, Axl is the more important Gas6R. To better clarify the relative contribution of the different Gas6Rs, we assessed the sensitivity of Gas6R-deficient mice to rescue with rGas6. Therefore, the effect of rGas6 in the acute hemolytic anemia model in $\mathrm{Axl}^{-/-}$mice was analyzed. Because our previous experiment showed that $\mathrm{PHZ}$ at the usual dosage was lethal for $A x^{-/-}$mice, we reduced the PHZ dose by $50 \%$ on days 0 and 1 . Mice received $2 \mu \mathrm{g} / \mathrm{d}$ of rGas 6 or vehicle $(0.9 \% \mathrm{NaCl})$. Consistent with a model whereby Axl would mediate the response to Gas6, rGas6 did not significantly improve the erythropoietic response in $\mathrm{Axl}^{-/-}$mice (Figure 4C). These findings suggest that the residual expression of Tyro3 and Mertk cannot compensate for the absence of Axl and that Axl is more important than the other 2 Gas6Rs.

Our data also showed that $A x l^{-/-}$mice were more sensitive to PHZ than Gas6 $6^{-/}$mice. However, because the genetic background

\section{Figure 4}

Expression of Gas6 and Gas6Rs in erythroblasts. (A) Western blot analysis of $\mathrm{Axl}$ and Mertk in spleen erythroblasts isolated from WT, Tyro3 ${ }^{--}, A x l^{--}$, and Mertk ${ }^{k d}$ mice. Equal amounts of protein cell lysates were loaded per well. (B) Erythropoiesis of Tyro3--- $\mathrm{Ax}^{\mathrm{H}^{--}}$or Mertk $\mathrm{Kd}^{\mathrm{d}}$ mice in response to PHZ. Overall genotypic differences versus WT (after Dunnett's correction for multiple testing) were as follows: Tyro3 ${ }^{-1-}$, $P=0.49$ ( $n=18) ; A x l^{--}, P=0.007(n=9) ;$ Mertk $^{k d}, P=0.97(n=5)$. (C) WT and $A x^{H^{--}}$mice were subjected to PHZ-induced hemolytic anemia (injections of a half dose of PHZ on days 0 and 1) and treated with saline or rGas6 (2 $\mu \mathrm{g}$ daily intraperitoneally). $P<0.05, A x t^{-/-}$and $A x^{H^{--}}$plus rGas6 versus WT; $P>0.05, A x^{H^{--}}$versus $A x^{-1-}$ plus rGas6.

of the $\mathrm{Gas}^{-/-}$mice and mice deficient in any one of the Gas6Rs is not identical, firm conclusions about the relative importance, when directly comparing these mutant mice, cannot be drawn. However, additional experiments revealed that the response to PHZ was comparable in Gas $6^{-/}$mice on mixed 50\% $129 / \mathrm{Sv} \times 50 \%$ Swiss (Figure 3A) and 100\% C57BL/6 genetic backgrounds (Supplemental Figure 1), indicating that differences in genetic backgrounds do not seem to determine, at least not in a major way, the magnitude of the erythropoietic response.

Release of Gas6 from erythroblasts in response to Epo. To assess whether the erythropoietic defect in Gas $6^{-/-}$mice is cell autonomous, we analyzed whether erythroblasts produce and release Gas6 in response to Epo. By quantitative real-time RT-PCR, we detected Gas6 mRNA transcript levels (normalized for GAPDH levels), but these remained unchanged in unfractionated spleens of WT mice 0 and 3 days after PHZ injection ( $0.89 \pm 0.24$ and $0.20 \pm 0.11$ copies $/ 10^{2}$ copies $G A P D H$, respectively; $\left.n=3 ; P>0.5\right)$. However, from day 3 to day 6 , with the generation of more splenic erythroblasts, Gas $6 \mathrm{mRNA}$ levels increased significantly to $5.81 \pm 3.48$ copies $/ 10^{2}$ copies GAPDH $(n=3 ; P=0.04)$. Furthermore, ELISA revealed that upon stimulation with Epo $(10 \mathrm{IU} / \mathrm{ml})$, isolated Ter- $119^{+}$splenic erythroblasts from WT mice released Gas6 into culture medium $(6 \pm 0.3 \mathrm{ng} / \mathrm{ml} / 24 \mathrm{~h}$ with Epo versus undetectable levels without Epo, $n=6$ ), whereas Gas6 was undetectable in the conditioned culture medium of Epo-stimulated Gas $6^{-/-}$erythroblasts. Western blotting confirmed detection of Gas6 protein in lysates of unstimulated UT7 cells (a human erythroid cell line; ref. 31) and that both the intracellular Gas 6 content and the amount of secreted Gas6 were dose-dependently increased by Epo (Figure 5).

Moreover, in WT mice, Gas6 plasma levels significantly increased during the erythropoietic response to hemolytic anemia (baseline, $5.5 \pm 1.3 \mathrm{ng} / \mathrm{ml}$; day 6 following PHZ, $9.1 \pm 1.3 \mathrm{ng} / \mathrm{ml} ; n=10$,

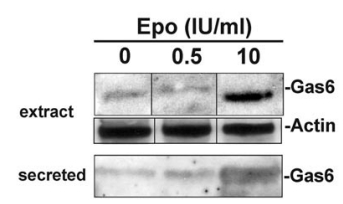

\section{Figure 5}

Release of Gas 6 from erythroblasts in response to Epo. Western blot analysis showing increased levels of Gas6 in cellular extracts and medium conditioned by UT7 human erythroid cells in response to $4 \mathrm{~h}$ stimulation with various concentrations of Epo. Total actin levels confirm the equal loading of cell protein extracts in each lane. Lanes showing Gas 6 in cellular extracts were run on the same gel but were noncontiguous, as indicated by black lines. 
A

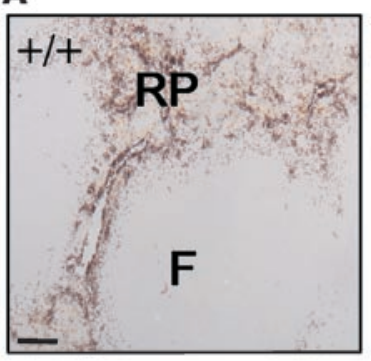

B

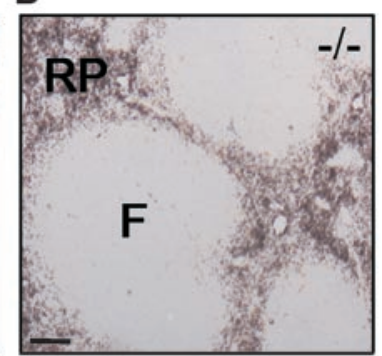

C
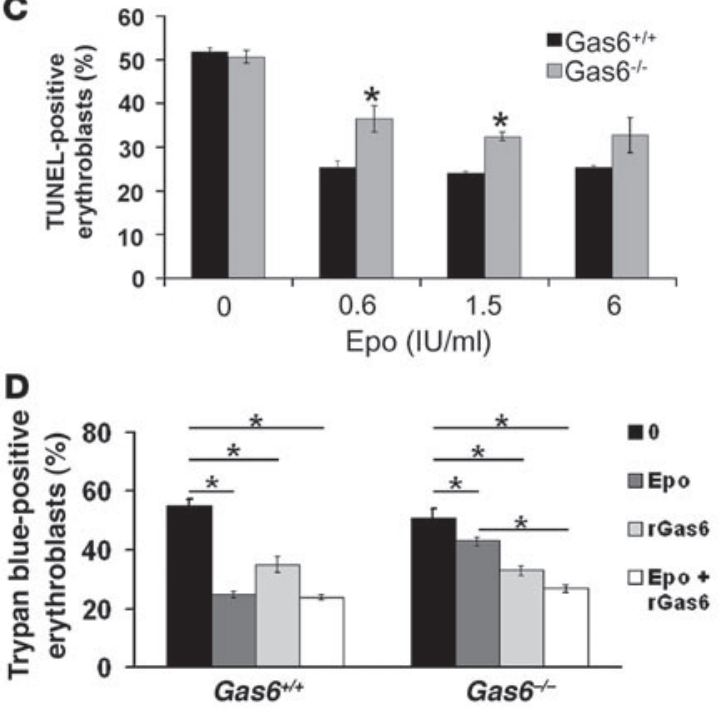

$P<0.05)$. Conversely, in $134.3 \mathrm{LC} \mathrm{Epo-TAg}^{\mathrm{H}}$ mice (32), a model with chronic anemia due to low Epo levels, plasma Gas6 levels were reduced to $44.0 \% \pm 0.1 \%$ of the plasma level in the corresponding WT mice $(n=6 ; P<0.05)$. Overall, these data indicate that Gas 6 and Epo are coordinately regulated and that erythroblasts themselves produce Gas 6 and release more Gas 6 when stimulated by Epo.

Gas 6 enhances the survival response of erythroblasts to Epo. We next sought to determine the mechanisms by which Gas 6 regulates erythropoiesis. We first excluded the possibility that the impaired erythropoietic response in anemic $\mathrm{Gas}^{-/-}$mice was attributable

\section{Figure 6}

Impaired Epo survival response of $\mathrm{Gas}^{-/-}$erythroblasts. (A and B) TUNEL staining of spleen 3 days after induction of hemolytic anemia by PHZ. Black staining with TUNEL denotes localized apoptotic cells in WT (A) and $\mathrm{Gas6}^{-1-}$ (B) spleen. F, follicle; RP, red pulp. Scale bars: $100 \mu \mathrm{m}$. (C) TUNEL assay of WT and $\mathrm{Gas6}^{-1-}$ erythroblasts isolated 6 days after $\mathrm{PHZ}$ injection. Cells were cultured for $16 \mathrm{~h}$ in the presence or absence of recombinant human Epo at the indicated concentrations. The TUNEL assay was quantified by flow cytometry $(n=4)$; results are expressed as the percentage of apoptotic erythroblasts, cells doublestained for TUNEL and Ter-119. (D) Dead cell count of splenic erythroblasts (Trypan blue ${ }^{+}$cells) collected on day 6 after $\mathrm{PHZ}$ injection and maintained in culture for $24 \mathrm{~h}$ in the presence or absence of Epo (1.5 $\mathrm{IU} / \mathrm{ml})$ or Epo plus human rGas6 $(400 \mathrm{ng} / \mathrm{ml}) . n=8$ per group. Values are mean \pm SEM. ${ }^{*} P<0.05$ versus WT.

to a deficiency in iron or Epo production (Supplemental Notes 3 and 4). We then evaluated whether the erythropoietic defect in Gas $6^{-/}$mice was caused by an impaired response of their erythroblasts to the survival activity of Epo. Because Gas6 reduces apoptosis of fibroblasts (20), diminished survival of erythroblasts in Gas $6^{-/}$mice might indeed account for the suboptimal erythropoietic response in these mice. This hypothesis was further suggested by experiments indicating that the red pulp of spleens contained comparably low TUNEL ${ }^{+}$apoptotic cells in both genotypes under baseline conditions, but more apoptotic cells in Gas6 $6^{-1-}$ than WT mice at 3 (Figure 6, A and B) and 6 (data not shown) days after PHZ-induced hemolysis.

To analyze directly whether survival of $\mathrm{Gas}^{-/-}$erythroblasts in response to Epo is impaired, we isolated spleen erythroblasts at day 6 after PHZ injection. When cultured for 16 hours in the absence of Epo, approximately $50 \%$ of the erythroblasts of either genotype were apoptotic (Figure 6C). However, the survival response to exogenous Epo was different in WT versus Gas $6^{-/}$mice. Indeed, a low concentration of Epo sufficed to lower the number of WT apoptotic cells, from $52 \% \pm 0.7 \%$ in controls to $25 \% \pm 1.1 \%$ in cells treated with $0.6 \mathrm{IU} / \mathrm{ml}$ Epo (Figure 6C). In contrast, a similar concentration of Epo decreased the number of apoptotic Gas $6^{-1-}$ cells from $51 \% \pm 1.1 \%$ in controls to $36 \% \pm 2.1 \%$ in cells treated with $0.6 \mathrm{IU} / \mathrm{ml}$ Epo (Figure 6C). Maximal protection of Gas6 $6^{-/}$erythroblasts from apoptosis was achieved with a minimum of $1.5 \mathrm{IU} / \mathrm{ml}$

\section{Figure 7}

Gas6 is required for adhesion of erythroblasts. (A) Adherence of WT versus Gas6 ${ }^{-1-}$ erythroblasts to fibronectin $(n=6)$, a mechanism essential for proliferation, survival, and expansion of these cells. The adhesion defect of Gas6 $^{-/-}$ erythroblasts was corrected by the addition of rGas6. (B) Survival response to Epo of WT and $\mathrm{Gas6}^{-1-}$ erythroblasts adherent to fibronectin $(n=3)$. Apoptotic adherent erythroblasts stained by DAPI were counted as cells with nuclear fragmentation. (C) Expression of VLA4 and Ter-119 in WT $(n=9)$ and Gas6 $^{-/-}(n=8)$ spleen determined by flow cytometry. VLA4 expression is proportional to Ter-119 expression. (D) Adherence of WT versus $\mathrm{Gas6}^{-/-}$erythroblasts to fibronectin in the presence of $0.9 \% \mathrm{NaCl}$ (vehicle), LY294002, or anti-VLA4 antibody $\left(2 \mu \mathrm{g} / 10^{6}\right.$ cells) $(n=6)$. Values are mean \pm SEM. ${ }^{*} P<0.05$. Data were reproduced in $100 \%$ C57BL/6 background (not shown).
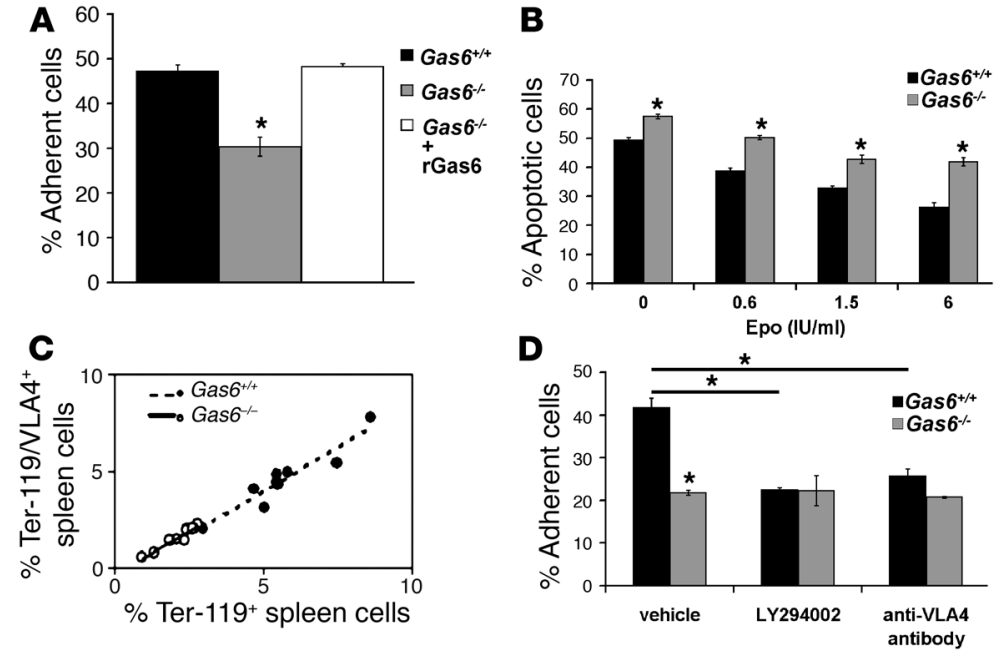

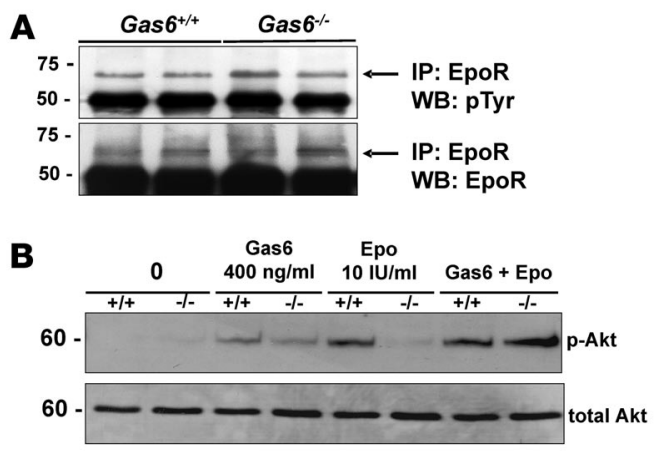

Epo, and this was still not sufficient to suppress apoptosis to the levels observed with WT erythroblasts (Figure 6C).

These findings were further confirmed and extended by using an independent method: counting dead erythroblasts after staining them with Trypan blue dye. At a concentration of $1.5 \mathrm{IU} / \mathrm{ml}$, Epo induced a maximal survival response in WT erythroblasts $(P<0.001)$; the same dose of Epo also exhibited a survival response in $\mathrm{Gas}^{-/}$erythroblasts $(P=0.02)$, but the response was significantly less prominent than with the WT erythroblasts, indicating that Gas 6 was required for a maximal response to Epo (Figure 6D). When tested alone, rGas 6 significantly enhanced erythroblasts survival in both genotypes $(P<0.001$ versus corresponding baseline controls). Although the protection against apoptosis by rGas 6 was not as effective as Epo alone in WT cells, Gas6 itself provided substantial survival signals for erythroblasts. Indeed, addition of

\section{Figure 8}

Epo signaling in WT and Gas6 ${ }^{-/-}$erythroblasts. (A and B) Western blot analysis of erythroblasts isolated from spleen 6 days after $\mathrm{PHZ}$ treatment from WT and Gas6 $^{-/-}$mice. (A) Total and tyrosine-phosphorylated (pTyr) EpoR expression in WT and Gas6-/- erythroblasts. (B) Akt phosphorylation in response to Gas6 alone (400 ng/ml), Epo alone (10 IU/ $\mathrm{ml}$ ), or a combination of both. Densitometric quantification of the amount of tyrosine-phosphorylated EpoR and total EpoR revealed a comparable ratio of pTyr-EpoR to EpoR in both genotypes (not shown).

\section{Figure 9}

Relative cytokine levels in plasma and BM-derived macrophage releasate in WT and Gas6 $^{-/}$mice. A cytokine antibody array was performed in a pool of plasma of 8 mice in steady-state condition $(\mathbf{A})$ and a pool of releasate of 8 macrophage culture samples (B). Relative cytokine expression is shown in AU. Cytokines known to inhibit erythropoiesis are indicated in red. 1, Axl; 2, B lymphocyte chemoattractant; 3, CD40; 4, CD80L; 5, CD80T; 6, cytokine responsive gene-2; 7, cutaneous T cell-attracting chemokine; $8, \mathrm{CXC}$-chemokine ligand-16; 9 , eotaxin; 10, eotaxin-2; 11, FasL; 12, fractalkine; 13, G-CSF; 14, GM-CSF; 15, IFN- $\gamma$; 16, IGF-binding protein 3 (IGFBP-3); 17, IGFBP-5; 18, IGFBP-6; 19, IL-10; 20, IL-12p40/p70; 21 , IL-12p70; 22, IL-13; 23, IL-17; 24, IL-1 $\alpha$; 25, IL-1 $\beta ; 26$, IL-2; 27, IL-3; 28, IL-3R $\beta$; 29, IL-4; 30, IL-5; 31, IL-6; 32, IL-9; 33, mouse IL-8 ortholog (KC or Gro-alpha); 34, leptin; 35, leptin receptor; 36 , LPS-induced CXC chemokine; 37 , L-selectin; 38 , lymphotactin; 39, monocyte chemotactic protein-1 (MCP1); 40, MCP5; 41, M-CSF; 42, monokine induced by IFN $\gamma$; 43, macrophage inflammatory protein $1 \alpha$ (MIP1 $\alpha)$; 44, MIP1 $\gamma ; 45$, MIP2; 46, MIP3 $\alpha$; 47, MIP3 $\beta$; 48, platelet factor 4; 49, P-selectin; 50, RANTES; 51 , SCF; 52 , SCF $1 \alpha ; 53$, soluble TNF-receptor 1 (sTNF-R1); 54, sTNF-R2; 55, thymus activation regulated chemokine; 56 , T cell activation gene $3 ; 57$, thymus expressed chemokine; 58 , tissue inhibitor of metalloproteinase $1 ; 59$, TNF- $\alpha$; 60, thrombopoietin; 61, VCAM-1; 62, VEGF.
A
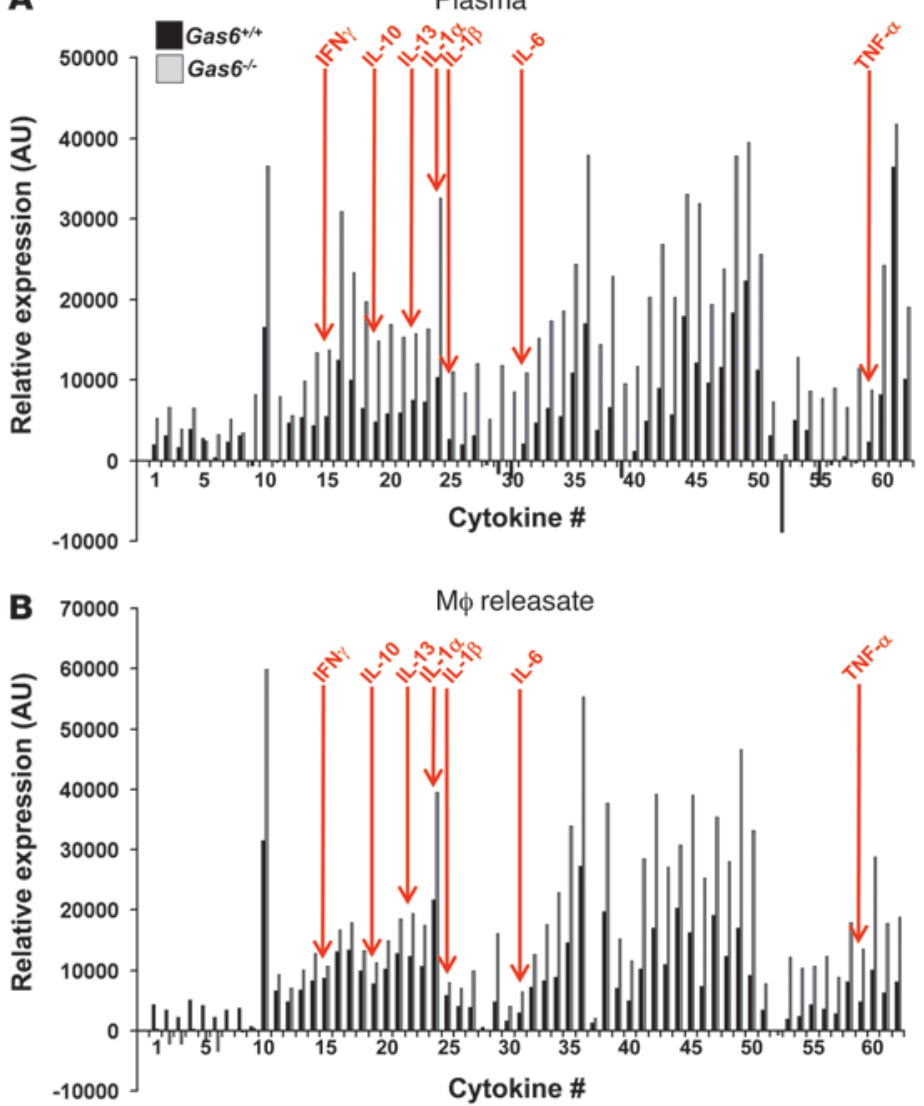

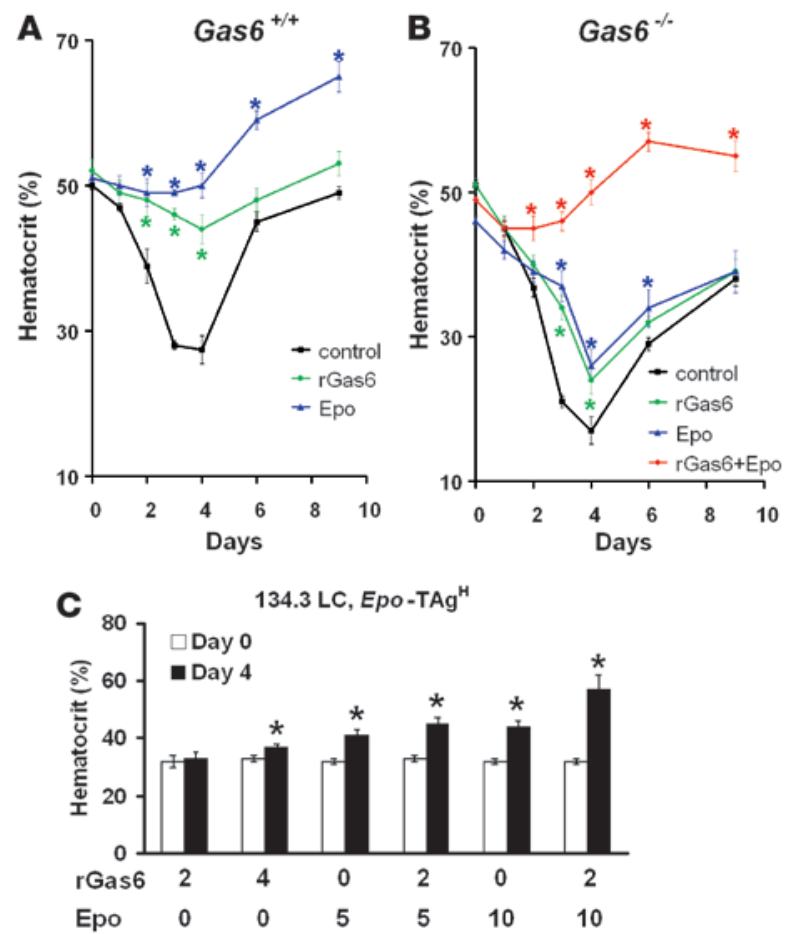

In addition, we compared the survival response to Epo of WTand $\mathrm{Gas}^{-/-}$-adherent erythroblasts. More WT- than $\mathrm{Gas}^{-/-}$-adherent erythroblasts survived in response to Epo (reduction of apoptotic cells, WT, 50\%; Gas6-/-, 30\%; Figure 7B). Similar findings were obtained when using Gas $6^{-/}$mice on mixed 50\% 129/Sv $\times 50 \%$ Swiss and $100 \%$ C57BL/ 6 genetic backgrounds (data not shown). However, the magnitude of the genotypic difference in survival response was not more notable when culturing the cells on fibronectin (Figure 7B) than in suspension (Figure 6C).

We then studied in more detail why adhesion of erythroblasts to fibronectin was impaired in the absence of Gas6. We observed that $\mathrm{Gas}^{-1-}$ and WT erythroblasts expressed comparable levels of VLA $4 / \alpha_{4} \beta_{1}$ integrin (Figure $7 C$ ), raising the question of whether downstream signaling (which promotes VLA4 activation) was impaired. Indeed, adhesion in the presence of anti-VLA4 antibodies was reduced in WT but not in Gas $6^{-/-}$erythroblasts, suggesting that absence of Gas6 impaired VLA4 activation (Figure 7D). In addition, the PI3K inhibitor LY294002 impaired the adhesion of WT and $\mathrm{Gas}^{-/-}$erythroblasts to the same extent as the anti-VLA4 antibody (Figure 7D). Thus, Gas6 favors PI3K and VLA4 activation.

Gas6, alone and together with Epo, activates the Akt survival pathway. The finding that Gas 6 regulated the response to Epo suggested a possible role of Gas6 in the signaling induced by Epo. By immunoblotting, we found that the expression levels of Epo receptor (EpoR) in splenic erythroblasts, isolated at day 6 after PHZ treatment, were comparable in WT and $\mathrm{Gas}^{-/-}$mice (Figure 8A). Tyrosine phosphorylation of EpoR was also not different between genotypes (Figure 8A), indicating that absence of Gas6 did not impair the activation of EpoR in erythroblasts and thus suggesting that the defect may affect more downstream signaling steps. As signal transduction of Epo in erythroblasts and of Gas 6 in other cell types is known to involve the activation of PI3K and its downstream target, Akt (20,21,36-39), we assessed whether absence of Gas 6 affected the activation of Akt in erythroblasts.

\section{Figure 10}

Therapeutic potential of rGas6 in acute and chronic anemia. (A and B) WT (A) and Gas6 $^{-1-}$ mice (B) were subjected to PHZ induced hemolytic anemia (injections on day 0 and 1 ) and treated with saline (control), human rGas6 ( $2 \mu \mathrm{g}$ daily intraperitoneally), recombinant human Epo (10 IU every second day intraperitoneally), or a combination of rGas6 and Epo. Overall genotypic differences relative to the control of the same genotype (calculated after Bonferroni correction for multiple testing) were as follows: rGas6-treated WT, $P<0.001$; Epo-treated WT, $P<0.001$; rGas6-treated Gas6-/- $P=0.004$; Epo-treated Gas6-/-, $P=0.008$; rGas6- and Epo-treated Gas6 ${ }^{-/}, P<0.001$. (C) Hematocrit levels in transgenic Epo-TAgH mice, a model of stable chronic anemia, treated with rGas6 alone or in combination with Epo at the indicated doses (in $\mu \mathrm{g}$ and IU, respectively). Values are mean $\pm \operatorname{SEM}(n=6)$. ${ }^{\star} P<0.05$ versus control, standard $t$ test.

As shown in Figure 8B, treatment of WT splenic erythroblasts with rGas6 alone $(400 \mathrm{ng} / \mathrm{ml})$ slightly stimulated phosphorylation of Akt, indicating that Gas6 itself was capable of initiating this survival pathway. A comparable, although smaller, effect was detected upon treating $\mathrm{Gas}^{-/-}$erythroblasts with rGas6. We therefore tested whether a higher dose of rGas6 restored Akt activation in Gas $6^{-/-}$ cells. Increasing the dose of rGas6 did not increase Akt phosphorylation, indicating that maximal activation was reached with a rGas6 concentration ranging $200-400 \mathrm{ng} / \mathrm{ml}$. This suggests that other signaling pathways are defective in $\mathrm{Gas}^{-/-}$erythroblasts.

Cotreatment of WT erythroblasts with Epo and rGas6 together also resulted in a strong Akt phosphorylation signal, which was comparable to the signal induced by Epo alone. The lack of a synergistic effect of rGas6 and Epo in WT cells is likely attributable to the fact that WT erythroblasts already release saturating levels of Gas6. In contrast, cotreatment of $\mathrm{Gas}^{-/-}$erythroblasts with Epo and rGas 6 together induced a much stronger Akt phosphorylation signal than treatment with Epo or rGas6 alone. In fact, Akt phosphorylation in $\mathrm{Gas}^{-/}$erythroblasts after cotreatment with Epo plus rGas6 was comparable to the levels observed in WT erythroblasts treated with Epo alone. Thus, apart from stimulating the Akt survival pathway itself, Gas6 also synergized with EpoR signaling to activate the Akt survival pathway.

Gas6 dampens the release of erythroid-inhibitory factors by macrophages. Gas6 and its cognate receptors Tyro3, Axl, and Mertk are also expressed by macrophages (40). Because erythroblasts proliferate, differentiate, and enucleate within the erythroblastic island, which includes a central macrophage (reviewed in ref. 41), we also sought to determine whether Gas6, via a paracrine effect, regulates the release of erythroid-inhibitory factors from macrophages. We therefore analyzed the expression of various cytokines in the plasma and supernatant of BM-derived macrophages of $\mathrm{Gas}^{-/-}$mice using a mouse protein cytokine array. This analysis revealed that loss of Gas6 increased the expression of several cytokines known to inhibit erythropoiesis (i.e., IL-10, IL-13, IL-1 $\alpha$, IL-1 $\beta$, IL-6, and TNF- $\alpha$; Figure 9) (42). Thus, in addition to its autocrine activity on the erythroblast (reinforcing Epo signaling), Gas6 also seemed to regulate erythropoiesis indirectly in a paracrine loop by regulating the release of erythroid-inhibitory factors from macrophages in the erythroblastic island.

Treatment of mouse models of acute and chronic anemia with rGas6. Prompted by the finding that Gas 6 regulated the erythropoietic response to anemia, we evaluated whether administration of rGas6 to WT mice would improve the erythropoietic response to anemia 
A

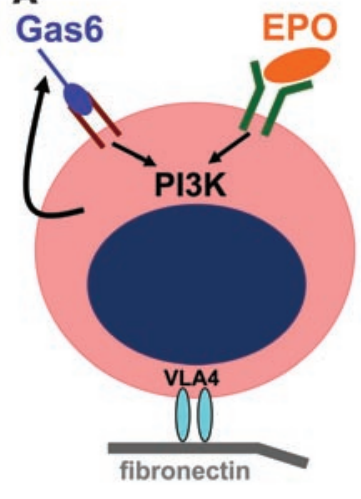

B

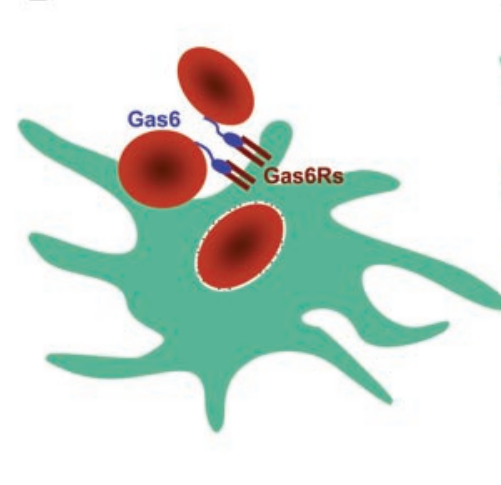

C

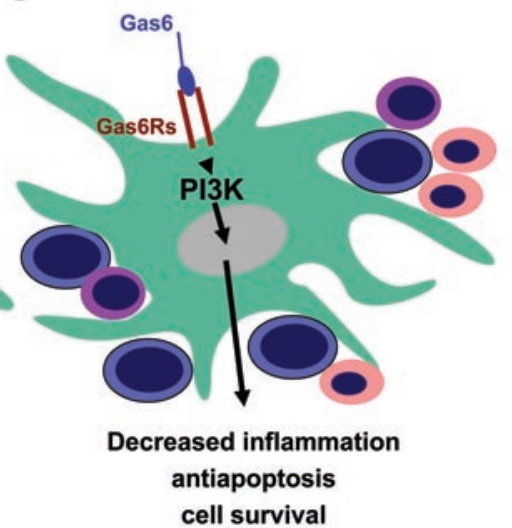

Figure 11

Model summarizing the role of the Gas6 pathway in erythropoiesis. (A) Gas6 released by erythroblasts in response to Epo interacts with Gas6R on the cell surface, leading to signaling for cell survival and possible cell proliferation, maturation, and differentiation, and enhances EpoR signaling by activating PI3K and its effector Akt in these cells (autocrine effect). In addition, Gas 6 favors adhesion of erythroblasts to fibronectin via VLA4/ $\alpha_{4} \beta_{1}$ integrin activation. (B) Gas 6 acts as a bridging molecule between senescent rbc and Gas 6 Rs, driving engulfment of the bound senescent rbc. (C) Interaction of Gas6 and Gas6Rs downregulates the release of erythroid-inhibitory factors from macrophages in the erythroblastic island.

using 2 models of acute anemia (i.e., hemolytic anemia induced by PHZ and anemia induced by blood loss) as well as 1 transgenic mouse model of chronic anemia (see below and Supplemental Note 5). In each model, the magnitude of the therapeutic effect of rGas 6 was compared with that of Epo. We first determined whether rGas 6 or Epo could prevent the development of PHZ-induced hemolytic anemia by delivering these compounds from day 0 , when $\mathrm{PHZ}$ injections were initiated (prevention trial). Upon administration of Epo or rGas6 to WT mice, hematocrit levels failed to drop after induction of hemolysis, indicating that both Epo and rGas6 protected these mice against the development of anemia (Figure 10A). Unlike Epo, rGas6 did not increase hematocrit levels to supraphysiologic levels from day 6 onward (Figure 10A). Compared with the control, the reticulocyte counts at 3 and 6 days after PHZ were increased by Epo (control, $9 \% \pm 1 \%$ and $30 \% \pm 2 \%$; Epo, $31 \% \pm 4 \%$ and $50 \% \pm 4 \% ; n=6 ; P<0.001)$ as well as by rGas6 $(21 \% \pm 2 \%$ and $35 \pm 3 \% ; n=6 ; P<0.05$, calculated by repeated measurements statistics). Epo stimulated the reticulocytosis to higher levels than rGas6 $(P<0.05)$.

The therapeutic potential of rGas6 to stimulate erythropoiesis in WT mice in response to acute anemia was not restricted to the PHZ model, but was also detected in a bleeding model of acute anemia. In these experiments, we administered Epo or rGas6 from day 2, 1 day after the last bleeding. Thus, unlike the prevention trial in the PHZ model, we used the bleeding model to examine whether we could restore the hematocrit levels once the mice were already anemic (regression trial). Administration of Epo restored hematocrit levels by day 6 and significantly increased the hematocrit levels even to supraphysiological levels (Supplemental Figure 2). Delivery of rGas6 also accelerated the erythropoietic recovery, although less rapidly than Epo, and not to supraphysiologic levels (Supplemental Figure 2). Taken together, rGas6 induced a therapeutic effect in both a prevention and a regression trial.

We also analyzed whether delivery of rGas6 was capable of increasing the hematocrit levels in a mouse model of chronic anemia. As very few mouse models of chronic anemia exist and a model of chronic anemia caused by a relative resistance to Epo was unavailable, we used a transgenic mouse strain (134.3 LC, Epo-TAg $\left.{ }^{\mathrm{H}}\right)$ that has low Epo levels and develops chronic anemia (hematocrit levels of $30 \%$, compared with $50 \%$ in WT) (32). This model has been previously validated for testing of erythropoietic therapies (43). Administration of 5 or 10 IU Epo every other day for 4 days increased relative hematocrit levels by $28 \%$ and $36 \%$, respectively $(P<0.001$; Figure 10C). Thus, although Epo was capable of stimulating erythropoiesis in this model of chronic anemia, its erythropoietic activity was less potent than in the acute anemia models. Consistent herewith, rGas6 also induced more modest effects. A daily dose of $2 \mu \mathrm{g}$ rGas 6 was ineffective, while a dose of $4 \mu \mathrm{g}$ rGas 6 increased the relative hematocrit levels by $12 \%$, a statistically significant effect (Figure 10C). Thus, in 3 different models of anemia, delivery of rGas6 induced an erythropoietic effect.

Treatment of Epo-resistant anemia with rGas6. The above findings suggested that Epo requires Gas6 for an optimal response. Considering the growing medical need to develop alternative strategies to treat patients with relative Epo resistance, we assessed whether rGas6 amplifies the therapeutic potential of Epo. To try to mimic the clinical situation, we used 2 models characterized either by impaired Epo responsiveness (Gas $6^{-/-}$mice) or by low Epo levels (134.3 LC, Epo-TAg ${ }^{\mathrm{H}}$ mice).

After PHZ, and compared with untreated controls, Gas $6^{-/-}$mice had a similar and significant erythropoietic response to $\mathrm{rGas} 6$ or Epo alone (Figure 10B). These responses, when compared with the corresponding and more prominent rises in identically treated WT mice (Figure 10A), indicated that $\mathrm{Gas}^{-/-}$mice were relatively resistant to administration of Epo or rGas6 (Figure 10B). For example, in WT mice, Epo yielded an absolute rise in hematocrit of $21 \%$ and $23 \%$ at 3 and 4 days after PHZ, respectively, whereas increases in Gas $^{-/-}$mice were $16 \%$ and $13 \%$. Similarly, rGas6 caused an absolute increase in hematocrit in WT mice of $23 \%$ and $17 \%$ at days 3 and 4 , respectively, compared with $9 \%$ and $7 \%$ increases in $\mathrm{Gas}^{-/-}$ mice. The dampened response to Epo in $\mathrm{Gas}^{-/-}$mice provides further evidence that Gas6 enhances the activity of Epo, while the 
suboptimal response to rGas 6 probably reflects the depleted stores of endogenous Gas6 in $\mathrm{Gas}^{-1-}$ mice that could not be restored by systemic delivery of the protein.

Notably, and providing additional support for the cooperative function of Gas 6 and Epo in acute anemia, combined administration of rGas 6 and Epo synergistically stimulated erythropoiesis in the PHZ-induced anemic Gas $6^{-/-}$mice. Indeed, compared with Epo and rGas6 treatment alone, hematocrit levels were higher after cotreatment with Epo and rGas6 (Figure 10B). Reticulocyte counts in $\mathrm{Gas}^{-/-}$mice were also higher after combined treatment with Epo and rGas6 together than after administration of each factor alone (reticulocyte counts at days 3 and 6 after PHZ, rGas6 plus Epo, $35 \% \pm 4 \%$ and $55 \% \pm 7 \%$; rGas6, $16 \% \pm 1 \%$ and $16 \% \pm 2 \%$; Epo, $10 \% \pm 1 \%$ and $19 \% \pm 3 \% ; n=6 ; P<0.001)$. Similar findings were obtained when using $\mathrm{Gas}^{-/-}$mice on mixed $50 \% 129 / \mathrm{Sv} \times 50 \%$ Swiss and $100 \%$ C57BL/ 6 genetic backgrounds (data not shown). Thus, rGas6 amplified the therapeutic effect of Epo.

When using the 134.3 LC Epo-TAg $^{\mathrm{H}}$ mouse, a model of chronic anemia caused by reduced Epo levels, delivery of Epo alone (10 IU) increased relative hematocrit levels by $36 \%$ (see above). However, when rGas6 and Epo were coadministered, hematocrit levels increased by $78 \%$, much higher than those obtained with administration of either drug alone (Figure 10C). Thus, in preclinical models of both Epo resistance and Epo insufficiency, rGas6 amplified the therapeutic activity of Epo.

\section{Discussion}

The most important finding of our study is that Gas 6 plays a critical role in the generation of erythroid progenitors and erythroblasts, in part by enhancing their survival response to Epo. As a result, absence of Gas 6 impairs the erythropoietic response and hematocrit recovery upon induction of acute anemia. Conversely, treatment with rGas6 stimulates hematocrit recoveries and protects WT mice against the development of anemia, induced by hemolysis or in part by blood loss. Moreover, in preclinical models of Epo resistance (Gas6 $6^{-/-}$mice) or Epo insufficiency (134.3 LC Epo-TAg ${ }^{\mathrm{H}}$ mice), rGas6 amplified the therapeutic effect of Epo to increase hematocrit levels in conditions of acute and chronic anemia. These genetic, pharmacologic, and molecular insights warrant further investigation of the therapeutic potential of Gas 6 for the treatment of anemia, particularly in the setting of Epo resistance.

To the best of our knowledge, a role for Gas 6 in erythropoiesis has not been reported previously. Gas 6 and its receptors were known to be expressed in the BM (12-15), but only conflicting effects on hematopoietic progenitors were documented thus far $(12,23)$. Here, we identified a role for Gas6 in the generation of sufficient numbers of erythroid progenitors, i.e., in the absence of Gas6, adult mice had fewer erythroblasts. The isolated erythroblast culture experiments revealed that Gas6 stimulates the survival of these cells, not only on its own but also by amplifying the response to Epo. Similar findings were obtained when we studied the survival Akt pathway. PI3K/Akt signaling is well known to mediate the survival activity of various growth factors (44) and was already previously shown to mediate the survival response to Gas6 in nonerythroblast cell types (20). We now show that Gas6 also activated Akt, alone and in combination with Epo, in erythroblasts. Furthermore, administration of rGas 6 alone to $\mathrm{Gas}^{-/-}$mice also provided some protection against the development of PHZinduced anemia. Thus, our physiological, cellular, and molecular studies consistently revealed the involvement of Gas6 in erythro- blast survival. These findings do not establish Akt signaling as the sole mechanism of action of Gas6. Indeed, we also observed that Gas6 allowed erythroblasts to adhere to fibronectin. Adhesion to the extracellular matrix is essential for the proliferation, survival, and expansion of erythroid progenitors and precursors as well as for the terminal stages of erythroid differentiation (33-35). In the present study, we found that this adhesion process was mediated, at least in part, by PI3K and VLA4 activation. Thus, Gas6, through activation of its receptors, appears to affect PI3K and integrin function in erythroblasts (Figure 11A), as we have previously documented for platelets (45).

Gas6 might also influence erythropoiesis via alternative mechanisms, independently of erythroblasts. For instance, we observed that Gas $6^{-1-} \mathrm{rbc}$ had a longer lifespan than did WT rbc and that the number of senescent rbc increased in $\mathrm{Gas}^{-/-}$mice compared with WT mice, implicating Gas6 in the clearance of senescent $\mathrm{rbc}$ by macrophages (Figure 11B). A similar role for Gas 6 has been documented for apoptotic cells (46). When cells become apoptotic or senescent, phosphatidylserine is exposed on their surface; by binding to phosphatidylserine on one side and to the Gas6R on the other side, Gas 6 forms a bridge between dying cells and macrophages, expressing Gas6Rs, which then engulf and clear these dying cells.

Moreover, Gas6 is known to downregulate the expression of inflammatory cytokines such as TNF- $\alpha$ by macrophages (reviewed in ref. 40). Because apoptotic depletion of BM progenitors by TNF- $\alpha$ has been implicated in the development of anemia (47), Gas6 might also play a beneficial role in erythropoiesis by reducing TNF- $\alpha$ production by macrophages (Figure 11C). Here we found that loss of Gas6 in BM-derived macrophages resulted in an increase, under steady-state conditions, in the production of several cytokines known to inhibit erythropoiesis (i.e., IL-10, IL-13, IL- $1 \alpha$, IL-1 $\beta$, IL- 6 , and TNF- $\alpha$ ).

The role of Gas6 in erythropoiesis appears to be cell autonomous: erythroblasts produce their own Gas6, they release Gas6 upon activation by Epo, and the released Gas 6 then binds to Axl on the same or neighboring erythroblasts and amplifies the survival activity of Epo (Figure 11A). In addition, Gas6 favors erythroblast adhesion via PI3K and VLA4. Gas6 has a similar intrinsic role in blood platelets, which release their Gas6 stores upon activation by platelet agonists and thereby amplify the platelet aggregation response to these agonists (24). The advantage of such a system is that the effector cell itself (i.e., erythroblast or platelet) is capable of enhancing its own sensitivity to the primary stimulus (i.e., Epo or platelet agonists), thereby amplifying the biological response. Obviously, apart from an autocrine role for Gas6 in erythroblasts, Gas6 may also be released by neighboring cells in hematopoietic tissues. For example, Gas6 secreted by macrophages in the spleen or BM may play a paracrine role (Figure 11, B and C). All 3 Gas6Rs were expressed on isolated erythroblasts and were capable of binding Gas6, but Axl played a prominent role in erythropoiesis. It remains to be determined why Tyro3 and Mertk play apparently negligible roles and whether this relates to the lack of an appropriate intracellular signaling machinery for these receptors in erythroblasts.

One might expect that the survival defect of the erythroid progenitors would cause reduced hematocrit levels under baseline conditions in $\mathrm{Gas}^{-/-}$mice. Two reasons might explain why this did not occur: (a) the increased lifespan of the mature rbc compensated for this defect; and (b) the defect was too subtle to affect the low level of erythropoiesis in baseline conditions, but sufficient 
to impair the intense erythropoietic response to anemia. Indeed, regardless of the stimulus (i.e., blood loss or hemolysis), hematocrit levels were lower and reticulocytosis was impaired, requiring a longer recovery time to restore the hematocrit levels in Gas $6^{-/-}$ mice. The defect was even more dramatic in $A x l^{-/-}$mice, resulting in life-threatening reductions in hematocrit levels (i.e., below 11\%) and causing demise of more than half of the mutant mice. Moreover, rGas6 failed to improve the erythropoietic response in these mice, while WT mice displayed an improvement under similar conditions. These data indicate that the residual expression of Tyro3 and Mertk cannot compensate for the absence of Axl and that Axl is more important than the other 2 Gas6Rs in erythroblasts. The more predominant role of Axl compared with Tyro3 and Mertk might relate to their relative affinities for Gas6, which vary more than 100-fold, with Axl having a higher affinity than Tyro3 and both in turn higher than Mertk (17).

Differences in genetic background between $\mathrm{Gas}^{-/-}$and $\mathrm{Axl^{-/- }}$ mice (see Methods) did not determine in a major way the magnitude of the erythropoietic response because responses to $\mathrm{PHZ}$ were comparable in $\mathrm{Gas}^{-1-}$ mice on pure $100 \% \mathrm{C} 57 \mathrm{BL} / 6$ and mixed $50 \%$ $129 / \mathrm{Sv} \times 50 \%$ Swiss backgrounds. One possible explanation, therefore, why loss of Axl resulted in a more severe phenotype than loss of Gas6, could relate to the fact that Axl may bind another ligand than Gas6. Protein S might be such a candidate, as it has previously been proposed to bind to Axl (reviewed in ref. 48). Unfortunately, to the best of our knowledge, mice lacking protein $S$ do not exist to allow us to test the in vivo role of protein $S$ in erythropoiesis in response to $\mathrm{PHZ}$. In addition, Axl has been proposed to induce biological responses, independently of Gas6, via mechanisms involving homophilic interactions of Axl-expressing cells (49).

How relevant a player is Gas6 in erythropoiesis? At first sight, one might argue that the fact that loss of Epo, but not of Gas6, results in embryonic lethality $(24,25)$ would imply that Gas 6 is redundant. When compared side by side, in absolute numbers, Epo induced a stronger erythropoietic response than did Gas6 in WT mice after PHZ and in 134.3LC Epo-TAg ${ }^{\mathrm{H}}$ mice as well as stronger activation of Akt in isolated WT erythroblasts. Nonetheless, Gas6 entirely normalized the hematocrit levels in WT mice and was equipotent to Epo in Gas $6^{-/-}$mice after PHZ; moreover, Gas6 directly promoted the survival of isolated erythroblasts. Perhaps even more relevant is the finding that Gas 6 amplified the response to Epo. This was most evident when analyzing the hematocrit levels induced in response to acute anemia in $\mathrm{Gas}^{-/-}$mice (a model of Epo resistance) and in the 134.3LC Epo-TAg ${ }^{\mathrm{H}}$ mice (a model of chronic anemia due to Epo insufficiency). Furthermore, whereas Epo regulates embryonic and adult erythropoiesis, Gas6 is more important for switching on erythropoiesis in pathological conditions in adulthood and thus may also be an attractive therapeutic target (see below). Considering that the role of Gas6 might be more important in disease than in normal health, it will be interesting to examine whether abnormal Gas 6 signaling contributes to the development of anemia associated with renal disease, cancer, arthritis, and other common disorders. Conversely, our findings, in combination with overexpression data of Gas6Rs in myeloproliferative disorders (15), raise the additional intriguing question as to whether activation of the Gas6 pathway also contributes to disease phenotypes characterized by too many circulating rbc (i.e., the myeloproliferative syndrome polycythemia vera).

What are the medical implications of our findings? Anemia is a common complication of many diseases, and, while Epo has been the drug of choice in the past $(7,8)$, resistance to Epo is becoming an increasing problem for numerous patients. In addition, optimal treatment with Epo is inconvenient in that it is generally administered several times per week, must be given intravenously or subcutaneously, and requires careful dose management to avoid polycythemia leading to thrombosis (11). The present findings that Gas6, alone or in combination with Epo, increased hematocrit levels in 3 models of acute anemia and 1 model of chronic anemia therefore warrant further study of its therapeutic potential. Overall, these findings provide insight into the molecular mechanisms by which Gas 6 promotes erythropoiesis and highlight its potential clinical importance in the treatment of anemia.

\section{Methods}

Mice. Gas $6^{-/}$and WT mice were progeny of the original colony, with a genetic background of 50\% $129 / \mathrm{Sv} \times 50 \%$ Swiss (24), and backcrossed onto C57BL/6 background for more than 10 generations. Tyro3 $3^{--}, A x l^{-/}$, and Mertk ${ }^{k d}$ mice were progeny of the original colony on a $50 \% 129 / \mathrm{Sv} \times 50 \%$ C57BL/ 6 background (50); control WT mice had the same genetic background. The 134.3 LC, Epo-Tag ${ }^{\mathrm{H}}$ heterozygous mice were progeny of the original colony on a pure C57BL/6 background (32). Animal experiments were approved by the Ethical Committee for Animal Experiments of the Medical Faculty of the University of Geneva, the Veterinary Service of Canton de Vaud, and the Institutional Animal Care and Research Committee of the University of Leuven.

Immunohistochemistry. Spleen and liver paraffin sections were stained for Ter-119 (BD Biosciences - Pharmingen). Apoptosis was evaluated by TUNEL labeling. ABC Vectastain kit (Vector) with DAB-cobalt-nickel staining was used to localize $\mathrm{TUNEL}^{+}$cells.

Flow cytometry. Single-cell suspensions were obtained from BM, spleen, or E13.5 embryo liver. Ammonium chloride lysis of rbc was performed. BM and spleen cells $\left(10^{6}\right)$ were incubated with rat anti-mouse CD16/CD32 to block nonspecific binding to $\mathrm{Fc}$ receptors. Cell suspensions were then stained with PE-conjugated anti-Ter119 antibodies and/or FITC-conjugated anti-VLA4 antibodies (BD Biosciences - Pharmingen) For FACSTUNEL, cells were fixed in $2 \%$ paraformaldehyde, permeabilized in $0.1 \%$ Triton X-100, and incubated with Terminal Transferase and FITC-dUTP (Roche Diagnostics) according to the manufacturer's instructions. Cell surface expression was analyzed using a Beckton Dickinson FACScan instrument and CellQuest software.

Colony formation assays. Single-cell suspensions of adult BM or E13.5 embryo liver were mixed with MethoCult M3434 (StemCell Technologies), plated in $35-\mathrm{mm}$ dishes, and cultured at $37^{\circ} \mathrm{C}, 5 \% \mathrm{CO}_{2}$. Colonies including BFU-E were scored at day 7. For CFU-E assay, cells were cultured in MethoCult 3230 containing murine Epo (R\&D Systems), and colonies were scored at day 3 .

Hematocrit and reticulocyte count. Blood was collected from the inferior vena cava or the retrobulbar plexus. Hematocrit was measured on blood drawn into a capillary tube. Reticulocyte counts were performed manually on blood smears stained with New Methylene Blue (Sigma-Aldrich). The reticulocyte index, assuming a normal hematocrit of $45 \%$, equals reticulocyte count $(\%) \times($ hematocrit/45).

In vivo biotinylation of rbc and annexin $V$ binding. $\mathrm{rbc}$ were biotinylated in vivo by an intravenous infusion of N-hydroxysuccimide biotin (NHS-biotin; Boehringer Mannheim) as previously described (51). In vivo biotinylation procedure resulted in essentially all the rbc being biotinylated, as judged by staining with streptavidin Red 670 (Invitrogen) $24 \mathrm{~h}$ after NHS-biotin infusion. Senescent rbc were detected by flow cytometry $30 \mathrm{~d}$ after NHS-biotin infusion as cells labelled with both biotin (detected by streptavidin Red 670) and FITC-conjugated annexin V (which binds phosphatidylserine; ref. 26). 
Engulfment of rbc by BM-derived macrophages. Macrophages were derived from BM precursors in the presence of M-CSF for 1 week. We added $30 \%$ of supernatant of the L929 cell line to cell culture medium (IMDM; Invitrogen) as a source of M-CSF. rbc isolated from peripheral blood were exposed to $3 \mathrm{mM} \mathrm{PHZ} \mathrm{in} \mathrm{PBS} \mathrm{for} 1 \mathrm{~h}$ at $37^{\circ} \mathrm{C}$, because $\mathrm{PHZ}$ induces lipid peroxidation and thereby phosphatidylserine translocation from the inner to the outer membrane surface (28). Cells were then washed in PBS, incubated with serum for $30 \mathrm{~min}$, and resuspended in IMDM to reach hematocrit of $50 \%$. This rbc suspension was incubated with adherent macrophages for $3 \mathrm{~h}$ at $37^{\circ} \mathrm{C}$. Adherent macrophages were washed with PBS, and noninternalized rbc were lysed by incubation in distilled water for $20 \mathrm{~s}$. Macrophages were covered with IMDM and examined in phase-contrast illumination.

Human $r$ Gas6. rGas6 was produced in the HEK293 line stably transfected with the pSecTagHygro expression vector encoding the human Gas6 cDNA. rGas6 was purified from conditioned medium with a modified calcium affinity chromatographic procedure similar to that reported for other $\gamma$-carboxyglutamic containing proteins (52).

Models of hemolytic anemia. Anemia was induced in mice by $\mathrm{PHZ}$ as described previously (53). We injected 2 or 3 doses of $0.5 \mathrm{mg} / 10 \mathrm{~g}$ body wt intraperitoneally as indicated (first dose on day 0 in the evening, second dose on day 1 in the morning, third dose on day 3 in the morning). For rescue experiments, mice received rGas6 or recombinant human Epo (Eprex; Janssen-Cilag) or a combination of both at the indicated concentrations. In a second model, autoimmune hemolytic anemia was induced by a single intraperitoneal injection of $200 \mu \mathrm{g}$ purified anti-rbc 34-3C IgG2a derived from unmanipulated NZB mice (29).

Measurement of Epo, Gas6 and Tyro3 levels. Epo concentrations were measured by ELISA (Quantikine IVD; R\&D Systems). Cross-reaction of the kit allowed detection of mouse Epo. The corrected absolute values of mouse Epo were determined as previously reported (43). Gas6 concentrations were measured by ELISA. Goat polyclonal antibody against Gas6 (AF986; R\&D Systems) was coated overnight at $4^{\circ} \mathrm{C}$ at $0.5 \mu \mathrm{g} / \mathrm{ml}$. Microtiter plates were then washed and blocked for $2 \mathrm{~h}$ with assay buffer (PBS plus $10 \%$ FCS). Murine rGas6 (986-GS; R\&D Systems) and samples were added after dilution in assay buffer. Plates were incubated overnight at $4^{\circ} \mathrm{C}$. After washing, the secondary biotinylated antibody (BAF986; R\&D Systems) was incubated for $45 \mathrm{~min}$. After washing, avidin peroxidase (BD Biosciences - Pharmingen) was added for $30 \mathrm{~min}$ at $37^{\circ} \mathrm{C}$. Sigma Fast OPD (Sigma-Aldrich) was used as a substrate. Color development was stopped with addition of $3 \mathrm{M} \mathrm{H}_{2} \mathrm{SO}_{4}$, and absorbance was measured at $492 \mathrm{~nm}$. The standard curve was linear between 0.2 and $1 \mathrm{ng} / \mathrm{ml}$ with a detection limit at $0.1 \mathrm{ng} / \mathrm{ml}$. Supernatants were diluted 1:10 and plasma samples 1:10 and 1:20. Gas6 levels were below the detection limit in Gas $6^{-1-}$ samples. Tyro3 concentration in erythroblast extracts was measured by ELISA (mouse Dtk ELISA; R\&D Systems).

Cell isolation, purification, and culture. Single-cell suspensions were obtained from spleen ( 6 days after PHZ injection) or BM. Ammonium chloride lysis of $\mathrm{rbc}$ was performed. Primary erythroblasts were isolated using MACS Ter-119 MicroBeads (Miltenyi Biotec) according to the manufacturer's instructions. Cells were stimulated with rGas6, recombinant human Epo (Eprex), or both at the indicated concentrations. For phospho-Akt and total Akt detection, the experiment was terminated by diluting the cells with ice-cold PBS containing $50 \mu \mathrm{M} \mathrm{NaVO}_{4}$. UT7 cells were maintained in DMEM containing $10 \%$ fetal bovine serum and $1 \mathrm{IU} / \mathrm{ml}$ Epo. Before each experiment, cells were starved in DMEM with $0.1 \%$ BSA overnight. Starved cells were then stimulated with the indicated concentrations of Epo. Stimulation was terminated by diluting the cells with ice-cold PBS containing $50 \mu \mathrm{M} \mathrm{NaVO}_{4}$ (37). Conditioned medium of UT7 cells was concentrated using Amicon Ultra (Millipore).
Immunoprecipitations and Western blotting. For immunoprecipitation, erythroblasts were lysed in the presence of the following protease inhibitors: $\mathrm{NaVO}_{4}, 1 \mu \mathrm{M}$; NaF, $1 \mathrm{mM}$; complete inhibitors (Roche Diagnostics). For detection of EpoR phosphotyrosine residues, the resulting supernatants were immunoprecipitated with an antibody directed against EpoR. Western blot analysis as performed using the following antibodies: antiRse (Tyro3), anti-Axl, anti-Mertk (Santa Cruz Biotechnology), anti-phosphotyrosine (clone 4G10; Upstate) and anti-murine EpoR (Upstate and R\&D Systems). For analysis of phospho-Akt and total Akt (PhosphoPlus Akt [Ser473] kit; Cell Signaling Technology), $100 \mu \mathrm{g}$ of lysate was resolved by SDS-PAGE and subjected to immunoblotting.

Adhesion of erythroblasts to fibronectin. Microtiter plates were coated with fibronectin (Sigma-Aldrich) and then blocked for $2 \mathrm{~h}$ at room temperature with BSA. Ter-119+ erythroblasts $\left(10^{5}\right)$ in RPMI supplemented with BSA $(2 \mathrm{mg} / \mathrm{ml})$ were added per well. Unattached cells were removed after $1 \mathrm{~h}$, and wells containing attached cells were washed twice with PBS. Control plates containing both unattached and attached cells were centrifuged, and supernatant was removed. Attached cells and controls (both attached and unattached cells) were stained with $0.2 \%$ crystal violet in $10 \%$ ethanol. The dye was eluted with $33 \%$ acetic acid, and the absorbance was measured at $570 \mathrm{~nm}$. The percent of adhesion to fibronectin was calculated by the optical density of attached erythroblasts divided by the optical density of controls (attached plus unattached erythroblasts). When indicated, adhesion experiments were performed in the presence of recombinant human Epo, LY294002 (Sigma-Aldrich), or anti-VLA4 antibody (clone PS/2; $2 \mu \mathrm{g} / 10^{6}$ cells) (54). Apoptotic adhering erythroblasts were identified by the fragmentation of their nucleus stained by DAPI (55).

Quantitative real-time RT-PCR. Gene expression of Gas6, Tyro3, Axl, and Mertk was quantified by real-time RT-PCR, relative to the expression of GAPDH (Gas6) or $\beta$-actin (Tyro3, Axl, and Mertk), using an ABI PRISM 7000 (Applied Biosystems) real-time cycler and the following primers: GAPDH forward, 5'-CAACGGGAAGCCCATCAC-3'; reverse, 5'-CGGCCTCACCCCATTTG-3'; Gas6 forward, 5'-CGAGTCTTCTCACACTGCTGTT-3'; reverse, 5'-GCACTCTTGATATCGTGGATAGAAATAC-3'; Tyro3 probe, 5'-CCTGGCCACTTCCCGACCAGC-3'; forward, 5'-GTGAAGCCCGCAACATAAAAG-3'; reverse, 5'-GGTGCTTGAAGGCGAACAAT-3'; Axl probe, 5'-AAGGGAGTCACCACCTCCCGCAC-3'; forward, 5'-TCATGTGAAGCCCACAATGC-3'; reverse, 5'-GGAGCACTGTGATGGTGGCT-3'; Mertk probe, 5'-GGAGCACTGTGATGGTGGCT-3'; forward, 5'-CGCCAAGGCCGCATT-3'; reverse, 5'-TCGGTCCGCCAGGCT-3'. For Gas6, each reaction contained $4 \mu \mathrm{l}$ SYBR Green PCR Master Mix (Applied Biosystems), $50-500 \mathrm{nM}$ primer pair, $5 \mu \mathrm{lDNA}$, and water to a final volume of $20 \mu \mathrm{l}$. PCR parameters were as follows: initial denaturation at $95^{\circ} \mathrm{C}$ for 10 min followed by 40 cycles of $15 \mathrm{~s}$ at $60^{\circ} \mathrm{C}$. Gas 6 mRNA transcript levels were normalized to GAPDH levels. For Tyro3, Axl, and Mertk, Taqman quantitative real-time PCR was performed with the Taqman Universal PCR master mix kit (Applied Biosystems). Gas6R mRNA transcript levels were shown as the number of copies of receptor per 1,000 copies of $\beta$-actin.

Cytokine antibody array. Expression of various cytokines in plasma and macrophage supernatant was investigated by using a mouse protein cytokine array (62 cytokines; RayBiotech). Array membranes were processed according to the manufacturer's instructions. We individually added $1 \mathrm{ml}$ of a plasma pool (8 mice) or supernatant to the membrane and incubated at room temperature for $2 \mathrm{~h}$. The results with immunoreactivity were assessed and quantified by using a VerSaDoc Imaging System (Bio-Rad).

Statistics. Data are represented as mean \pm SEM of $n$ determinations. Standard $t$ tests were used to calculate significance levels between groups. When analyzing experiments performed at multiple time points, ANOVA for repeated measurement statistics were used to calculate the overall significance and average difference between WT and Gas $6^{-/}$mice, followed 
by standard $t$ tests to calculate differences at the individual time points. Because repeated measurement statistics cannot handle missing values, we assigned the lowest hematocrit level (i.e., 11\%) to mice that died in the course of the experiment. To compare multiple genotypes (e.g., Tyro3 $3^{-/}, A x^{-/-}$, or Mertk ${ }^{k d}$ ) against the WT control group, we applied a Dunnett's correction for multiple testing, using the WT group as reference. To compare the different treatments with each other (e.g., Gas6, Epo, or the combination of both), we applied Bonferroni corrections to correct for multiple testing. We used SPSS (Statistical Software Package version 11.0 for Mac OS X) for all these calculations. A $P$ value less than 0.05 was considered significant.

\section{Acknowledgments}

We thank Giuseppe Celetta, Solange Charvier, Lena Kieckens, Sandra Jansen, Nathalie Flores, and Jorge Ritz for technical assistance and Catherine Lacombe (Hôpital Cochin, Paris, France) for providing UT7 cells. This work was supported by the Dinu Lipatti-Dr. Henri Dubois-Ferrière Foundation and Swiss National
Foundation for Scientific Research grants 3100-064027.00, 3234066307.01 (Marie-Heim-Vögtlin), 3232-066350.01 (SCORE), 3200066351.01, PP00B-106690/1, and 3100A0-105872. E.M. Conway was supported in part by the Belgian Federation Against Cancer. M. Tjwa and D. Lambrechts are research fellows of the IWT and FWO, respectively (Belgium). P. Carmeliet was supported by generous grants from the Bristol Myers Squibb Foundation Inc. (New York, New York, USA) and the GOA (KU Leuven, Belgium).

Received for publication September 18, 2006, and accepted in revised form November 7, 2007.

Address correspondence to: Anne Angelillo-Scherrer, Service and Central Laboratory of Hematology, CHUV, University Hospital Center and University of Lausanne, Rue du Bugnon 46, CH-1011 Lausanne, Switzerland. Phone: 41-21-314-42-22; Fax: 41-21-31441-80; E-mail: Anne.Angelillo-Scherrer@chuv.ch.
1. Weatherall, D.J., and Provan, A.B. 2000. Red cells I: inherited anaemias. Lancet. 355:1169-1175.

2. Richmond, T.D., Chohan, M., and Barber, D.L. 2005. Turning cells red: signal transduction mediated by erythropoietin. Trends Cell Biol. 15:146-155.

3. Koury, M.J., and Bondurant, M.C. 1990. Erythropoietin retards DNA breakdown and prevents programmed death in erythroid progenitor cells. Science. 248:378-381.

4. Krystal, G. 1983. A simple microassay for erythropoietin based on $3 \mathrm{H}$-thymidine incorporation into spleen cells from phenylhydrazine treated mice. Exp. Hematol. 11:649-660.

5. Blumenauer, B., Cranney, A., Clinch, J., and Tugwell, P. 2003. Quality of life in patients with rheumatoid arthritis: which drugs might make a difference? Pharmacoeconomics. 21:927-940.

6. Bohlius, J., et al. 2005. Recombinant human erythropoietin and overall survival in cancer patients: results of a comprehensive meta-analysis. J. Natl. Cancer Inst. 97:489-498.

7. Eschbach, J.W., et al. 1987. Correction of the anemia of end-stage renal disease with recombinant human erythropoietin. Results of a combined phase I and II clinical trial. N. Engl. J. Med. 316:73-78.

8. Winearls, C.G., et al. 1986. Effect of human erythropoietin derived from recombinant DNA on the anaemia of patients maintained by chronic haemodialysis. Lancet. 2:1175-1178.

9. Lacombe, C. 1996. Resistance to erythropoietin. N. Engl. J. Med. 334:660-662.

10. Italian Cooperative Study Group for rHuEpo in Myelodysplastic Syndromes. 1998. A randomized double-blind placebo-controlled study with subcutaneous recombinant human erythropoietin in patients with low-risk myelodysplastic syndromes. Br. J. Haematol. 103:1070-1074.

11. Locatelli, F., et al. 2004. Revised European best practice guidelines for the management of anaemia in patients with chronic renal failure. Nephrol. Dial. Transplant. 19(Suppl. 2):ii1-ii47.

12. Avanzi, G.C., et al. 1997. GAS6, the ligand of Axl and Rse receptors, is expressed in hematopoietic tissue but lacks mitogenic activity. Exp. Hematol. 25:1219-1226.

13. Crosier, P.S., Freeman, S.A., Orlic, D., Bodine, D.M., and Crosier, K.E. 1996. The Dtk receptor tyrosine kinase, which binds protein $\mathrm{S}$, is expressed during hematopoiesis. Exp. Hematol. 24:318-323.

14. Graham, D.K., et al. 1995. Cloning and developmental expression analysis of the murine c-mer tyrosine kinase. Oncogene. 10:2349-2359.

15. Neubauer, A., et al. 1994. Expression of axl, a transforming receptor tyrosine kinase, in normal and malignant hematopoiesis. Blood. 84:1931-1941.
16. Manfioletti, G., Brancolini, C., Avanzi, G., and Schneider, C. 1993. The protein encoded by a growth arrest-specific gene (gas6) is a new member of the vitamin K-dependent proteins related to protein $\mathrm{S}$, a negative coregulator in the blood coagulation cascade. Mol. Cell. Biol. 13:4976-4985.

17. Nagata, K., et al. 1996. Identification of the product of growth arrest-specific gene 6 as a common ligand for Axl, Sky, and Mer receptor tyrosine kinases. J. Biol. Chem. 271:30022-30027.

18. Nakano, T., et al. 1997. Cell adhesion to phosphatidylserine mediated by a product of growth arrestspecific gene 6. J. Biol. Chem. 272:29411-29414.

19. Varnum, B.C., et al. 1995. Axl receptor tyrosine kinase stimulated by the vitamin K-dependent protein encoded by growth-arrest-specific gene 6 . Nature. 373:623-626.

20. Goruppi, S., Ruaro, E., and Schneider, C. 1996. Gas6, the ligand of Axl tyrosine kinase receptor, has mitogenic and survival activities for serum starved NIH3T3 fibroblasts. Oncogene. 12:471-480.

21. Goruppi, S., Ruaro, E., Varnum, B., and Schneider, C. 1999. Gas6-mediated survival in NIH3T3 cells activates stress signalling cascade and is independent of Ras. Oncogene. 18:4224-4236.

22. Saller, F., Burnier, L., Schapira, M., and AngelilloScherrer, A. 2006. Role of the growth arrest-specific gene 6 (gas6) product in thrombus stabilization. Blood Cells Mol. Dis. 36:373-378.

23. Dormady, S.P., Zhang, X.M., and Basch, R.S. 2000. Hematopoietic progenitor cells grow on $3 \mathrm{~T} 3$ fibroblast monolayers that overexpress growth arrestspecific gene-6 (GAS6). Proc. Natl. Acad. Sci. U. S. A. 97:12260-12265.

24. Angelillo-Scherrer, A., et al. 2001. Deficiency or inhibition of Gas6 causes platelet dysfunction and protects mice against thrombosis. Nat. Med. 7:215-221.

25. Wu, H., Liu, X., Jaenisch, R., and Lodish, H.F. 1995. Generation of committed erythroid BFU-E and CFU-E progenitors does not require erythropoietin or the erythropoietin receptor. Cell. 83:59-67.

26. Manodori, A.B., and Kuypers, F.A. 2002. Altered red cell turnover in diabetic mice. J. Lab. Clin. Med. 140:161-165.

27. Cohen, P.L., et al. 2002. Delayed apoptotic cell clearance and lupus-like autoimmunity in mice lacking the c-mer membrane tyrosine kinase. J. Exp. Med. 196:135-140.

28. Jain, S.K., and Subrahmanyam, D. 1976. Studies on lipids of red blood cells in phenylhydrazine-treated albino rats. Indian J. Biochem. Biophys. 13:155-157.

29. Fossati-Jimack, L., et al. 1999. High pathogenic potential of low-affinity autoantibodies in experimental autoimmune hemolytic anemia. J. Exp. Med.
190:1689-1696.

30. Morisaki, H., and Sibbald, W.J. 2004. Tissue oxygen delivery and the microcirculation. Crit. Care Clin. 20:213-223.

31. Komatsu, N., et al. 1991. Establishment and characterization of a human leukemic cell line with megakaryocytic features: dependency on granulocyte-macrophage colony-stimulating factor, interleukin 3, or erythropoietin for growth and survival. Cancer Res. 51:341-348.

32. Maxwell, P.H., et al. 1993. Identification of the renal erythropoietin-producing cells using transgenic mice. Kidney Int. 44:1149-1162.

33. Hamamura, K., et al. 1996. A critical role of VLA-4 in erythropoiesis in vivo. Blood. 87:2513-2517.

34. Patel, V.P., and Lodish, H.F. 1987. A fibronectin matrix is required for differentiation of murine erythroleukemia cells into reticulocytes. J. Cell Biol. 105:3105-3118.

35. Yanai, N., Sekine, C., Yagita, H., and Obinata, M. 1994. Roles for integrin very late activation antigen-4 in stroma-dependent erythropoiesis. Blood. 83:2844-2850.

36. Bao, H., et al. 1999. Protein kinase B (c-Akt), phosphatidylinositol 3-kinase, and STAT5 are activated by erythropoietin (EPO) in HCD57 erythroid cells but are constitutively active in an EPO-independent, apoptosis-resistant subclone (HCD57-SREI cells). Blood. 93:3757-3773.

37. Bouscary, D., et al. 2003. Critical role for PI 3-kinase in the control of erythropoietin-induced erythroid progenitor proliferation. Blood. 101:3436-3443.

38. Haseyama, Y., et al. 1999. Phosphatidylinositol 3 -kinase is involved in the protection of primary cultured human erythroid precursor cells from apoptosis. Blood. 94:1568-1577.

39. Klingmuller, U., et al. 1997. Identification of a novel pathway important for proliferation and differentiation of primary erythroid progenitors. Proc. Natl. Acad. Sci. U. S. A. 94:3016-3021.

40. Lemke, G., and Lu, Q. 2003. Macrophage regulation by Tyro 3 family receptors. Curr. Opin. Immunol. 15:31-36.

41. Chasis, J.A. 2006. Erythroblastic islands: specialized microenvironmental niches for erythropoiesis. Curr. Opin. Hematol. 13:137-141.

42. Weiss, G., and Goodnough, L.T. 2005. Anemia of chronic disease. N. Engl. J. Med. 352:1011-1023.

43. Rinsch, C., et al. 2002. Delivery of erythropoietin by encapsulated myoblasts in a genetic model of severe anemia. Kidney Int. 62:1395-1401.

44. Takahashi, K., Murakami, M., and Yamanaka, S. 2005. Role of the phosphoinositide 3-kinase pathway in mouse embryonic stem (ES) cells. Biochem. Soc. Trans. 33:1522-1525. 
45. Angelillo-Scherrer, A., et al. 2005. Role of Gas6 receptors in platelet signaling during thrombus stabilization and implications for antithrombotic therapy. J. Clin. Invest. 115:237-246.

46. Scott, R.S., et al. 2001. Phagocytosis and clearance of apoptotic cells is mediated by MER. Nature. 411:207-211.

47. Papadaki, H.A., et al. 2002. Bone marrow progenitor cell reserve and function and stromal cell function are defective in rheumatoid arthritis: evidence for a tumor necrosis factor alpha-mediated effect. Blood. 99:1610-1619.

48. Hafizi, S., and Dahlback, B. 2006. Gas6 and protein S.
Vitamin K-dependent ligands for the Axl receptor tyrosine kinase subfamily. FEBS J. 273:5231-5244.

49. Heiring, C., Dahlback, B., and Muller, Y.A. 2004. Ligand recognition and homophilic interactions in Tyro3: structural insights into the Axl/Tyro3 receptor tyrosine kinase family. J. Biol. Chem. 279:6952-6958.

50. Lu, Q., et al. 1999. Tyro-3 family receptors are essential regulators of mammalian spermatogenesis. Nature. 398:723-728.

51. Dale, G.L., and Daniels, R.B. 1991. Quantitation of immunoglobulin associated with senescent erythrocytes from the rabbit. Blood. 77:1096-1099.
52. Yan, S.B. 1996. Review of conformation-specific affinity purification methods for plasma vitamin K-dependent proteins. J. Mol. Recognit. 9:211-218.

53. Lim, S.K., et al. 1998. Increased susceptibility in Hp knockout mice during acute hemolysis. Blood. 92:1870-1877.

54. Katayama, Y., et al. 2003. PSGL-1 participates in E-selectin-mediated progenitor homing to bone marrow: evidence for cooperation between E-selectin ligands and alpha4 integrin. Blood. 102:2060-2067.

55. Hasanbasic, I., Rajotte, I., and Blostein, M. 2005. The role of gamma-carboxylation in the anti-apoptotic function of gas6. J. Thromb. Haemost. 3:2790-2797. 